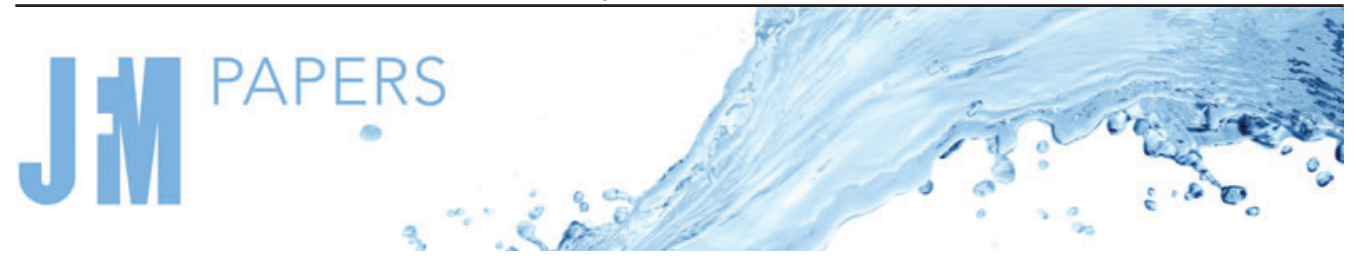

\title{
Organized kinetic energy backscatter in the hurricane boundary layer from radar measurements
}

\author{
Sydney Sroka ${ }^{1} \dagger$ and S.R. Guimond ${ }^{2}$ \\ ${ }^{1}$ Department of Mechanical Engineering, Massachusetts Institute of Technology, Cambridge, MA 02139, \\ USA \\ ${ }^{2}$ Joint Center for Earth Systems Technology (JCET) and Department of Physics, University of Maryland \\ Baltimore County, Baltimore, MD 21250, USA
}

(Received 2 November 2020; revised 1 July 2021; accepted 8 July 2021)

The fluid mechanics of hurricanes strongly depends on boundary layer energetics due to the warm-core nature of the system with peak velocities located at lower levels. One barrier that has inhibited a more complete characterization of energy transfer in the boundary layer is a lack of observations that resolve large, turbulent eddies. In particular, the occurrence and structure of upscale energy transfer (backscatter) in the hurricane boundary layer as well as the effects of backscatter on the vortex intensity are unknown. The analysis presented here of very high-resolution, three-dimensional wind observations from Hurricane Rita (2005) at peak intensity reveals large regions of organized backscatter in the boundary layer associated with coherent, turbulent eddies. Strong forwardscatter is also found next to the backscatter regions due to the interaction between adjacent eddies. Two components of the stress tensor are primarily responsible for this alternating scatter structure, as shown by large correlation coefficients between the fields: the radial-vertical component $\left(\tau_{13}\right)$ and the azimuthal-vertical component $\left(\tau_{23}\right)$ with average correlations of $79 \%$ and $49 \%$, respectively. The Leonard, Reynolds and cross-term stress components are also provided. The impact of the sub-filter-scale energy transfer is estimated by computing the kinetic energy budget for the resolved-scale and eddy-scale motions. The results show that the sub-filter-scale energy transfer term is of the same order as the other terms in the eddy-scale budgets, contributing between $16 \%$ and $40 \%$ to the local time tendency with an average contribution of approximately $30 \%$. These results indicate that the coherent turbulent eddies can affect the vortex dynamics through wave-wave nonlinear interactions, which can subsequently influence the wave-mean flow interactions. This is the first study to examine the full sub-filter-scale

$†$ Email address for correspondence: ssroka@mit.edu

(C) The Author(s), 2021. Published by Cambridge University Press. This is an Open Access article, distributed under the terms of the Creative Commons Attribution licence (http://creativecommons.org/ licenses/by/4.0/), which permits unrestricted re-use, distribution, and reproduction in any medium, provided the original work is properly cited. 


\section{S. Sroka and S.R. Guimond}

energy transfer and its impact on the kinetic energy budget in the hurricane boundary layer. These findings emphasize the importance of coherent turbulence in the energy cascade and have the potential to improve turbulence closure schemes used in numerical simulations.

Key words: turbulent boundary layers, vortex dynamics, atmospheric flows

\section{Introduction}

While large, turbulent eddies with wavelengths of the order of $1 \mathrm{~km}$ have been recognized as important in geophysical fluid dynamics for many years (Tennekes \& Lumley 1972; Stull 1988; Cushman-Roisin 1994), the study of these scales in very-large-Reynolds-number flows such as hurricanes has been limited. The primary reasons for this are: (i) a lack of turbulence-resolving measurements that probe deep into the boundary layer where turbulent kinetic energy (TKE) is the most significant, and (ii) computer resource limitations that inhibit the explicit calculation of turbulent eddies throughout the full hurricane domain, which can have radii of the order of hundreds of kilometres. Measurements of the hurricane boundary layer (HBL) over the open ocean are inherently difficult to make and have relied heavily on Lagrangian point samples made by small instruments dropped from aircraft ('dropsondes'; Franklin, Black \& Valde 2003). Dropsondes have been very useful for piecing together a picture of the HBL after compositing data over many hours and many storms, but the full, localized, turbulent structure of this layer cannot be studied with this dataset. Many years ago, the United States National Oceanic and Atmospheric Administration (NOAA) conducted flights into the heart of the HBL with manned research aircraft. However, after one aircraft nearly crashed into the ocean due to extreme turbulence (Marks et al. 2008), these manned flights were abandoned. Remote sensing is the only practical means by which to obtain complete, turbulence-resolving, three-dimensional measurements in the HBL. In regards to simulations, several recent studies have conducted large eddy simulations (LES) of hurricanes using a grid spacing of $30-60 \mathrm{~m}$ in the inner core region (approximately $50 \mathrm{~km}$ in radius) in order to fully resolve the most energetic, turbulent eddies (Bryan \& Rotunno 2009; Rotunno et al. 2009; Ito, Oizumi \& Niino 2017; Guimond, Sroka \& Proztko 2018a). However, large domain LES like these are currently far from routine, especially for operational hurricane models, which means that the effects of the eddies must be parameterized. Many mesoscale modelling studies, which use horizontal grid spacings of $1-2 \mathrm{~km}$, have documented a significant sensitivity of the hurricane intensity to changes in the turbulence parameterization, especially in the boundary layer (Smith \& Thomsen 2010; Bryan 2012; Kepert 2012). Managing the effects of this sensitivity requires an improved understanding of the fluid mechanics in the HBL from new measurements and new simulations to improve and evaluate turbulence parameterizations.

Commonly used turbulence parameterizations, such as the K-profile method, the Smagorinsky-Lilly scheme and the TKE method, are purely dissipative and assume that energy only transfers from larger scales to smaller scales (known as forwardscatter or direct energy cascade). It has been shown that energy can also transfer from smaller scales to larger scales (known as backscatter or inverse energy cascade) in simple, low-Reynolds-number flows (Kraichnan 1976; Leith 1990; Germano et al. 1991; Piomelli et al. 1991; Natrajan \& Christensen 2006), in the surface layer of atmospheric 
flows (Sullivan et al. 2003) and on the scales of convective clouds in hurricanes (Guimond et al. 2016). In addition, several studies have performed simulations of idealized airflow over a moving water surface, reminiscent of the air-sea interface, and found that energy can be transported from smaller to larger scales (Makin \& Kudryavtsev 1999; Rutgersson \& Sullivan 2005; Wang et al. 2020). However, the low Reynolds number of these simulations, very small domains focused on the air-sea interface and the idealized nature of the flow have limited applicability to the larger-scale (of the order of $1 \mathrm{~km}$ ) atmospheric characteristics of a mature HBL. Our focus in this paper is on these larger-scale turbulent structures with wavelengths of approximately $2 \mathrm{~km}$ that span at least the full depth of the HBL (Guimond et al. 2018b), which is typically approximately $1 \mathrm{~km}$. To the best of the authors' knowledge, there are no systematic studies of the full energy transfer on these scales in the HBL. This is partially because of a scarcity of high-resolution turbulence measurements in the HBL and because the few LES studies that are mentioned above have focused on the overall vortex intensity and the general flow structure of the HBL.

Backscatter can have important implications for the resolved-scale dynamics and the accuracy of large-scale predictions (Piomelli et al. 1991). Piomelli, Yu \& Adrian (1996) showed that omitting backscatter in an idealized flow can change whether perturbations to that flow grow or decay, and the amount of backscatter increased with the Reynolds number. Currently, it is unknown if backscatter exists in the HBL and what effect this might have on the localized dynamics or the overall vortex intensity. The backscatter process energizes the larger-scale motions, which can enhance the nonlinear interactions between waves and ultimately lead to increases in the mean vortex intensity. This pathway describes effects on the dynamics, but there are also feedbacks involving the thermodynamics, whereby backscatter can energize larger-scale motions that enhance the flux of enthalpy from the ocean to the atmosphere, possibly resulting in increased convective activity.

The main purpose of this paper is to characterize the energy transfer in the HBL over the open ocean from a set of very high-resolution radar measurements collected in the turbulent boundary layer of Hurricane Rita (2005) during an intense stage of the storm. These measurements reveal the ubiquitous presence of coherent turbulent eddies with scales of approximately $2 \mathrm{~km}$ that extend from the ocean surface, through the boundary layer and into the lower free atmosphere at a height of approximately $1.5 \mathrm{~km}$ (Guimond et al. (2018b); hereafter G18b). The role of the sub-filter-scale (SFS) energy transfer term in the kinetic energy (KE) budget is also examined. This is the first study to examine the full energy transfer term and its impact on the KE budget on the scales of large turbulent eddies in the HBL. Given the presence of coherent structures in the data, another purpose of this paper is to examine whether there is some organization or determinism in the SFS energy transfer that can be harnessed to improve turbulence parameterizations for coarse-grained models. Currently, some numerical models use stochastic forcing to represent the effects of under-resolved and un-resolved processes in the dynamics of various phenomena including hurricanes (Shutts 2005; Judt, Chen \& Berner 2016) and this process may not accurately reflect the physics of the HBL.

Section 2 describes the velocity observations from Hurricane Rita (2005) and the KE budget equation for our coordinate system and dynamic regime. Section 3 examines the components of the SFS energy transfer term to identify the features of the velocity fields which are primarily driving the backscatter. Section 4 compares all of the terms in the $\mathrm{KE}$ budget for both the resolved scale and eddy scale to explain the mechanism by which backscatter appears to be forcing the mean flow. Finally, $\S 5$ presents an idealized, 


\section{S. Sroka and S.R. Guimond}

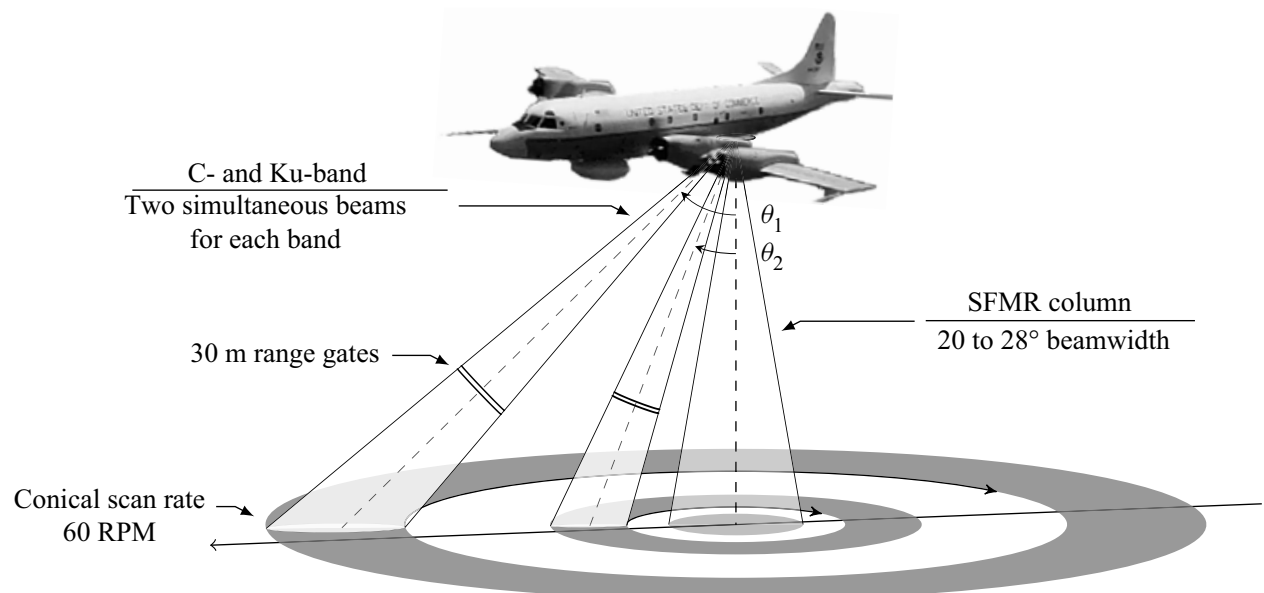

Figure 1. Conceptual diagram of the IWRAP instrument and scanning geometry onboard the NOAA P3 aircraft. The SFMR is another instrument designed to measure ocean surface winds.

conceptual model to explain the locations of the coherent forwardscatter and backscatter regions identified in the HBL.

\section{Methodology}

Analysing the energy transfer in the HBL depends on having very high-resolution velocity fields that can fully resolve turbulent eddies on scales of $1 \mathrm{~km}$. In a simulation study, Guimond et al. (2018a) showed that the Imaging Wind and Rain Airborne Profiler (IWRAP) instrument is able to fully resolve scales of $1 \mathrm{~km}$ and capture coherent turbulent eddies in the HBL. The IWRAP is a downward-pointing, conically scanning, dual-frequency (C- and Ku-band), dual-polarization, dual-beam airborne phased array radar that measures ocean surface returned radar power, volume reflectivity and Doppler velocity from precipitation. The IWRAP scans at 60 revolutions per minute, allowing wind retrievals with grid spacings of approximately $200 \mathrm{~m}$ and $30 \mathrm{~m}$ in the horizontal and vertical dimensions, respectively. The IWRAP is mounted on the NOAA WP-3D (P3) research aircraft, which performs radial transects through the storm centre at heights of $1.5-3.0 \mathrm{~km}$ above the surface. As a result, the mapped radar data are approximately in cylindrical coordinates $(r, \theta, z)$ with the storm centre at the origin. Figure 1 illustrates the sampling geometry of the IWRAP instrument on the P3 aircraft. The swath width (azimuth dimension) of the IWRAP data is approximately $1-2 \mathrm{~km}$ at the lower levels of the sampling volume.

In this study, IWRAP wind fields from an eyewall replacement cycle in Hurricane Rita (2005) are used to calculate and analyse the SFS energy transfer in the HBL, and also to evaluate the full KE budget. These wind fields have been thoroughly validated and compare very well to in situ flight level data (Guimond et al. (2014), G18b). For the data analysed here, the zonal and meridional velocity components have root mean square errors (RMSEs) of approximately $3.1 \mathrm{~m} \mathrm{~s}^{-1}$ and $3.8 \mathrm{~m} \mathrm{~s}^{-1}$, respectively, which is approximately $8 \%-10 \%$ of the sampled winds. The correlation coefficients of these velocity components with flight level data are very high at 0.99 . The vertical velocities are more difficult to measure and calculate, but still show good error statistics. After accounting for a 


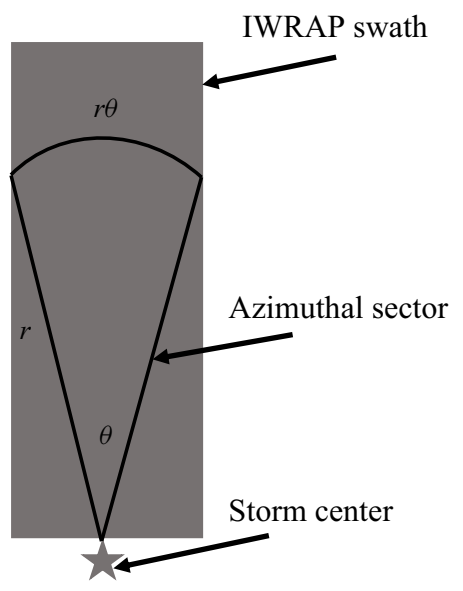

Figure 2. Schematic that illustrates the cylindrical coordinate system and IWRAP swath used to perform the azimuthal sector averaging and KE calculations. Note the IWRAP swath width is approximately $1-2 \mathrm{~km}$ wide at the lower levels.

small bias, the vertical velocity RMSEs are approximately $1.8 \mathrm{~m} \mathrm{~s}^{-1}$ with correlation coefficients of 0.65 . Furthermore, G18b compared the details of the eddy structures in the specific regions of each flight leg presented here to flight level data and showed that the eddies are captured well, including the vertical velocity component. Some regions of data have been removed because the signal quality was not sufficient. The uncertainty of the Doppler velocity measurements is proportional to the pulse-pair correlation coefficient and the number of pulses used in the average ( $n$ ) (Fernandez et al. 2005). The pulse-pair correlation coefficient varies between 0 and 1 with noise proportional to $1 / \sqrt{n}$; for the Rita data this corresponds to a value of approximately 0.1 after averaging 125 pulses. We have conducted sensitivity analysis on various thresholds and found that data above a pulse-pair correlation coefficient of 0.2 produce good quality wind fields in terms of validation with in situ data and relatively smooth variability with the surrounding wind field. This threshold has been applied to the data presented in this paper, but the amount of data removed was small and does not affect the analysis.

Before the energy transfer calculations are performed, the IWRAP velocity fields are pre-processed to remove grid-scale noise. A forward-backward box filter with a 2-point window in the $r$ direction and a 4-point window in the $z$ direction was applied. The three-dimensional wind fields from IWRAP are then azimuthally averaged across the swath into a two-dimensional, $r-z$ plane. This plane is approximately equal to a very small azimuthal sector mean of the full hurricane vortex. Figure 2 shows the cylindrical coordinate system for a typical IWRAP radial transect through the storm centre and the sector that is used to azimuthally average the data.

We calculate the SFS energy transfer using a methodology similar to those described in Leonard (1975), Piomelli et al. (1991), and Sullivan et al. (2003). A spatial filter $(G)$ is convolved with the total velocity fields $\left(u_{i}\right.$, where $i=1,2,3$ corresponds to the radial, tangential and vertical directions, respectively) in the radial direction to produce resolved-scale fields $\left(\tilde{u}_{i}\right)$ and SFS fields $\left(u_{i}^{\prime}\right)$ according to

$$
\begin{gathered}
\widetilde{u_{i}}(r, z)=\int u_{i}\left(r^{*}, z\right) G\left(r-r^{*}\right) \mathrm{d} r^{*} \\
u_{i}^{\prime}(r, z)=u_{i}(r, z)-\widetilde{u}_{i}(r, z) .
\end{gathered}
$$




\section{S. Sroka and S.R. Guimond}

The spatial filter used here is a $2 \mathrm{~km}$ forward-backward, box filter in physical space which avoids introducing phase shifts into the resolved-scale fields. We chose this filter length scale since many numerical simulations of hurricanes are conducted with a grid spacing of $1 \mathrm{~km}$, which means the smallest resolvable length scale is $2 \mathrm{~km}$. There is also a physical motivation for the filter length scale. In this paper, we have followed the definition of 'coherent structure' or 'coherent motion' from the review article on the subject by Robinson (1991) on p. 602: ' . . coherent motion is defined as a three-dimensional region of the flow over which at least one fundamental flow variable (velocity component, density, temperature, etc.) exhibits significant correlation with itself or with another variable over a range of space and/or time that is significantly larger than the smallest local scales of the flow'. In the study of G18b, auto-correlation and covariance analysis was performed on the IWRAP wind fields analysed here to detect the coherent structures and quantify the eddy characteristics, which revealed mean wavelengths of approximately $2 \mathrm{~km}$, horizontal wind speed perturbations of $10-20 \mathrm{~m} \mathrm{~s}^{-1}$, aspect ratios of approximately 1.3 and largely positive covariances between all velocity components. The dominant eddy wavelengths of approximately $2 \mathrm{~km}$ are much larger than the smallest local scales of the flow (approximately $200 \mathrm{~m}$ ) and thus, the eddies satisfy the 'coherent structure' definition described above. However, other definitions are possible, which may include additional constraints on the velocity field such as low pressure perturbations (Chong, Perry \& Cantwell 1990). While we anticipate that the large velocity perturbations identified in the data will also have disturbances in the pressure field, similar to what is shown in Marks et al. (2008), we do not have collocated pressure fields to perform a more thorough analysis.

Figure 3 shows KE spectra for the three radial passes of IWRAP data analysed in $\S 3$. The spectra are computed by removing the linear trend across the domain at each vertical level and performing Fourier transforms along the radial dimension. The spectra are then averaged over all heights since the turbulent structures are vertically coherent. Figure 3 shows secondary peaks in the $\mathrm{KE}$ at wavelengths from 1 to $3 \mathrm{~km}$, which represent the turbulent eddies under examination here. These scales are consistent with the auto-correlation and covariance analysis of G18b and motivates the chosen filter width of $2 \mathrm{~km}$. We will revisit this length scale choice in $\S 3$ where we describe the results of a sensitivity test that confirmed the overall structure is independent of the choice of filter length scale.

The three-dimensional, incompressible Navier-Stokes equation for the resolved-scale velocities is

$$
\frac{\partial \widetilde{u_{i}}}{\partial t}+\tilde{u}_{j} \frac{\partial \tilde{u_{i}}}{\partial x_{j}}=-g \delta_{i 3}-\frac{1}{\rho} \frac{\partial \tilde{p}}{\partial x_{i}}+v \frac{\partial^{2} \tilde{u_{i}}}{\partial x_{j} \partial x_{j}}-\frac{\partial \tau_{i j}}{\partial x_{j}},
$$

where $g$ is gravity, $\rho=\bar{\rho}(z)$ is an average density profile and $\tilde{p}$ is pressure. In this presentation for the KE, we have not included the Coriolis force in (2.3) because this term does no work. The molecular viscosity term in (2.3) can be neglected since the Reynolds number in a hurricane is extremely large, between $O\left(10^{9}\right)$ and $O\left(10^{12}\right)$ depending on whether the relevant length scale is the boundary layer depth or the radius of maximum wind. By taking the dot product of (2.3) with $2 \tilde{u_{i}}$, we arrive at the transport equation for the resolved-scale $\operatorname{KE}\left(\tilde{q}^{2}=\widetilde{u}_{i} \widetilde{u}_{i}\right)$

$$
\frac{\partial \tilde{q}^{2}}{\partial t}+\tilde{u}_{j} \frac{\partial \tilde{q}^{2}}{\partial x_{j}}=-2 g \delta_{i 3} \tilde{u}_{i}-\frac{2}{\rho} \tilde{u}_{j} \frac{\partial \tilde{p}}{\partial x_{j}}+2 \frac{\partial \tilde{u}_{i} \tau_{i j}}{\partial x_{j}}-2 \mathcal{P},
$$




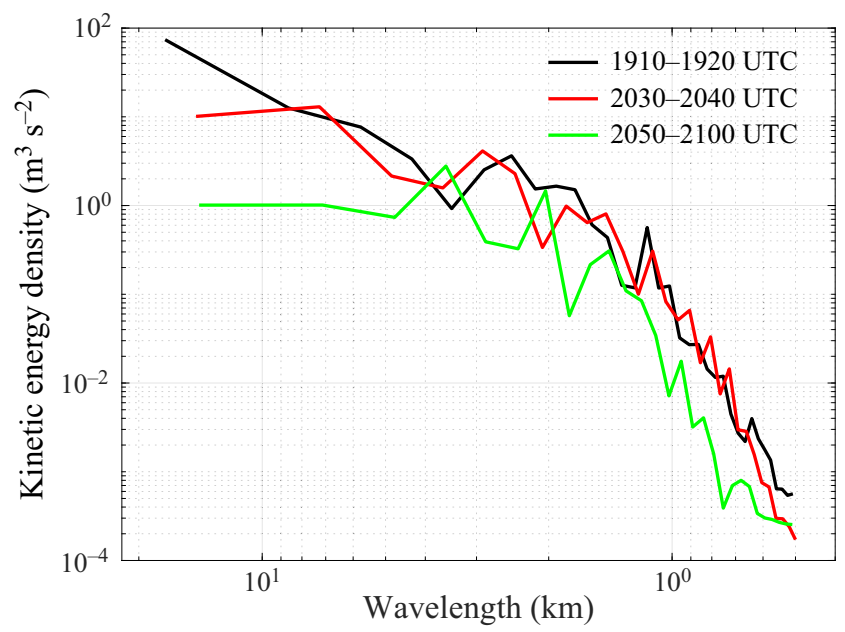

Figure 3. KE spectra for the three radial passes of IWRAP data analysed in this paper.

where $\mathcal{P}$ is the total production of SFS energy, defined as $\mathcal{P}=-\tau_{i j} \widetilde{S}_{i j}$. The two tensors which compose $\mathcal{P}$ are $\tau$, which is the flux tensor, and $\tilde{S}$, which is the strain rate tensor. Forwardscatter implies $\mathcal{P}>0$ and backscatter implies $\mathcal{P}<0$. The production term $-2 \mathcal{P}$ is also referred to as the SFS energy transfer term.

Before filtering the velocities into resolved-scale and SFS components, the data from an IWRAP swath are azimuthally averaged according to

$$
\bar{\phi}(r, z)=\frac{1}{r \theta} \int_{0}^{r \theta} \phi\left(r, \theta^{*}, z\right) r \mathrm{~d} \theta^{*}
$$

The expanded, azimuthally averaged KE transport equation in cylindrical coordinates is

$$
\begin{aligned}
\frac{\partial \tilde{\bar{q}}^{2}}{\partial t}+\underbrace{\tilde{\bar{u}} \frac{\partial \tilde{\bar{q}}^{2}}{\partial r}+\tilde{\bar{w}} \frac{\partial \tilde{\bar{q}}^{2}}{\partial z}=}_{\mathrm{ADV}} & -2 g \tilde{\bar{w}}-\underbrace{-\frac{2}{\rho}\left(\tilde{\bar{u}} \frac{\partial \tilde{\bar{p}}}{\partial r}+\tilde{\bar{w}} \frac{\partial \tilde{\bar{p}}}{\partial z}\right)}_{\text {PGF }}-2\left[\frac{1}{r} \frac{\partial r \tilde{\bar{u}} \tau_{r r}}{\partial r}-\frac{\tau_{\theta \theta} \tilde{\bar{u}}}{r}+\frac{\partial \tilde{\bar{u}} \tau_{r z}}{\partial z}\right. \\
& \left.+\ldots \frac{1}{r^{2}} \frac{\partial r^{2} \tilde{\bar{v}} \tau_{\theta r}}{\partial r}+\frac{\partial \tilde{\bar{v}} \tau_{\theta z}}{\partial z}+\frac{1}{r} \frac{\partial r \tilde{\bar{w}} \tau_{r z}}{\partial r}+\frac{\partial \tilde{\bar{w}} \tau_{z z}}{\partial z}\right]-2 \mathcal{P},
\end{aligned}
$$

where the azimuthally averaged, filtered radial, tangential and vertical velocities are $\tilde{\bar{u}}, \tilde{\bar{v}}$ and $\tilde{\bar{w}}$, respectively. Section 4 compares the relative magnitudes of the budget terms in (2.6); the terms are the time tendency of the resolved-scale KE, the advection term (denoted with ADV), the gravitational term, the pressure gradient force transport (denoted with PGF), the SFS energy transport term (denoted with square brackets) and the production term.

The flux tensor $\tau$ is the equal to the sum of the Leonard $\left(L_{i j}\right)$, Reynolds $\left(R_{i j}\right)$ and cross-term $\left(C_{i j}\right)$ stresses (or fluxes) as defined in Germano (1986), written here in 


\section{S. Sroka and S.R. Guimond}

summation notation for simplicity

$$
\begin{aligned}
& \tau_{i j}=\widetilde{\overline{u_{i}} \overline{u_{j}}}-\widetilde{\overline{u_{i}}} \widetilde{\widetilde{u_{j}}} \equiv L_{i j}+R_{i j}+C_{i j} \\
& L_{i j}=\widetilde{\widetilde{u_{i}}} \widetilde{\overline{u_{j}}}-\approx \widetilde{\widetilde{u_{i}}} \approx \widetilde{\overline{u_{j}}} \\
& R_{i j}=\widetilde{\overline{u_{i}^{\prime}} \overline{u_{j}}}-\widetilde{\bar{u}_{i}^{\prime}} \widetilde{\bar{u}_{j}^{\prime}} \\
& C_{i j}=\widetilde{\widetilde{u_{i}}}{\overline{u_{j}}}^{\prime}+\widetilde{\widetilde{u_{j}}} \overline{\bar{u}}_{i}^{\prime}-\widetilde{\widetilde{u_{i}}} \widetilde{\bar{u}_{j}^{\prime}}-\widetilde{\widetilde{u_{j}}}{\widetilde{\overline{u_{i}}}}^{\prime} .
\end{aligned}
$$

The Reynolds term represents interactions between SFS motions that project onto the resolved-scale motions, while the cross-term represents the direct interactions between SFS motions and the resolved scale. The residual between the flux tensor and the Reynolds and cross-terms is the Leonard term, which is a function of resolved-scale motions only. Finally, the strain rate tensor, in azimuthally averaged cylindrical coordinates, is

$$
\tilde{\bar{S}}=\frac{1}{2}\left[\begin{array}{ccc}
2 \frac{\partial \tilde{\bar{u}}}{\partial r} & \frac{\partial \tilde{\bar{v}}}{\partial r}-\frac{\tilde{\bar{v}}}{r} & \frac{\partial \tilde{\bar{u}}}{\partial z}+\frac{\partial \tilde{\bar{w}}}{\partial r} \\
\frac{\partial \tilde{\bar{v}}}{\partial r}-\frac{\tilde{\bar{v}}}{r} & \frac{\tilde{\bar{u}}}{r} & \frac{\partial \tilde{\bar{v}}}{\partial z} \\
\frac{\partial \tilde{\bar{w}}}{\partial r}+\frac{\partial \tilde{\bar{u}}}{\partial z} & \frac{\partial \tilde{\bar{v}}}{\partial z} & 2 \frac{\partial \tilde{\bar{w}}}{\partial z}
\end{array}\right] .
$$

\section{SFS energy transfer component breakdown}

The following subsections show individual radial passes of IWRAP data from Hurricane Rita (2005) and the associated energy transfer calculations in the boundary layer. During the time periods described below, the storm was undergoing an eyewall replacement cycle where an outer eyewall forms, contracts and merges together with the decaying, inner eyewall. On September 22, the inner eyewall was decaying and the outer eyewall was intensifying. Intense turbulence was located most prominently in the outer eyewall where strong vertical wind shear was present. On September 23, the outer eyewall contracted and merged with the inner eyewall shifting the location of the most intense turbulence to the inner edge of the merged eyewall. In the results described below, we first present the detailed structure of the total production of SFS energy $\mathcal{P}$ and the components that compose this quantity from a representative radial leg of data. Then, we show the robustness of these results with subsequent legs from a more general perspective.

\subsection{September 22 1910-1920 UTC pass}

This radial leg sampled the western portion of the storm during an eyewall replacement cycle. The inner and outer eyewall structures, as well as thin bands of radar reflectivity, located mostly on the inner edge of the outer eyewall, can be detected from this snapshot (see figures 4 and 6 in G18b). The azimuthally averaged, total velocity components for this pass, shown in figure 4 in cylindrical coordinates, reveal turbulent eddies in the radial region of the outer eyewall with characteristic wavelengths of approximately $2-2.5 \mathrm{~km}$. The black, dashed line in (a) identifies the $\bar{u}=0$ contour showing that the region below $z=1 \mathrm{~km}$ is dominated by inflow. The annotations indicate regions of forwardscatter $(\mathrm{F})$ and backscatter (B), and the white contour shows the boundary between forwardscatter and backscatter $(\mathcal{P}=0)$. The forwardscatter-backscatter pattern alternates regularly with 

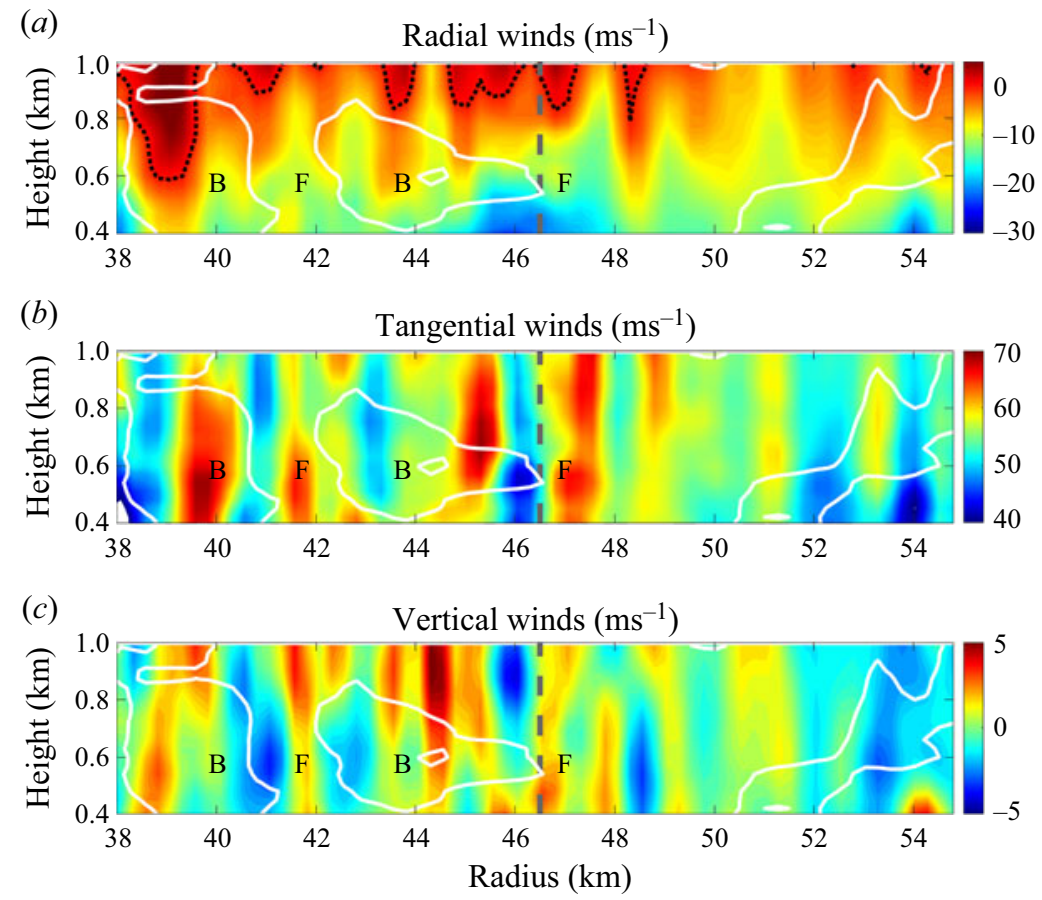

Figure 4. Nadir vertical cross-sections of the total, azimuthally averaged velocity components for the 1910-1920 UTC radial pass on 22 September 2005. The $x$-axis in all figures is the radial distance from the storm's centre. The annotations indicate regions of forwardscatter $(\mathrm{F})$ and backscatter (B), the black dashed contour line indicates where the radial velocity is zero and the white contour identifies where $\mathcal{P}=0$. The vertical dashed grey line near $r=46.5 \mathrm{~km}$ approximately identifies the radius of maximum wind of the secondary eyewall; the primary eyewall (not shown) is located near $r=26 \mathrm{~km}$.

a wavelength between 4 and $5 \mathrm{~km}$, which is approximately twice the wavelength of the eddies themselves (G18b).

The total production of SFS energy is shown in figure 5 with the same annotations as figure 4 . The production term is normalized by $u_{*}^{3} / y_{*}$, where $u_{*}$ is the average characteristic eddy velocity of $15 \mathrm{~m} \mathrm{~s}^{-1}$ and $y_{*}$ is the characteristic eddy length scale of $2 \mathrm{~km}$ (Guimond et al. 2018b). While to the best of the authors' knowledge no data at a similar Reynolds number are available for comparison, hopefully these scale factors will facilitate comparisons between these results and future studies.

To better understand how the organized and alternating structure of $\mathcal{P}$ aligns with the boundary layer eddies, we trace the features of $\mathcal{P}$ through the components of $\tilde{\bar{S}}$ and $\tau$, back to features of the velocity field. We start by considering the six unique components $\mathcal{P}_{i j}$, shown in figure 6 . Throughout this analysis, we will calculate the Pearson correlation coefficient $c$ between $\mathcal{P}$ and each component of $\mathcal{P}_{i j}, \widetilde{S_{i j}}, \tau_{i j}, L_{i j}, R_{i j}$ and $C_{i j}$, to serve as a quantitative metric of how much influence a given component has on $\mathcal{P}$. In figure 6 , it is clear from the correlation coefficients, the observed structure and the relative magnitudes that $\mathcal{P}_{13}$ and $\mathcal{P}_{23}$ (and their symmetric counterparts) largely govern the structure of $\mathcal{P}$. This indicates that the radial-vertical interactions and, to a lesser extent, the azimuthal-vertical interactions in $\tau$ and/or $\tilde{\bar{S}}$ are driving the SFS energy production. 


\section{S. Sroka and S.R. Guimond}

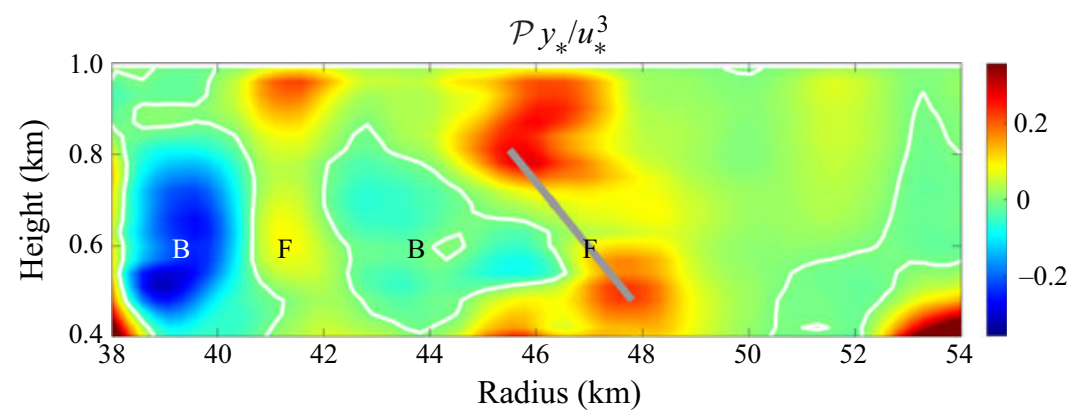

Figure 5. The total SFS energy for the 1910-1920 UTC pass. The grey line indicates a region of forwardscatter that is tilted with respect to the vertical and the other details on the figure are the same as in figure 4.

(a)
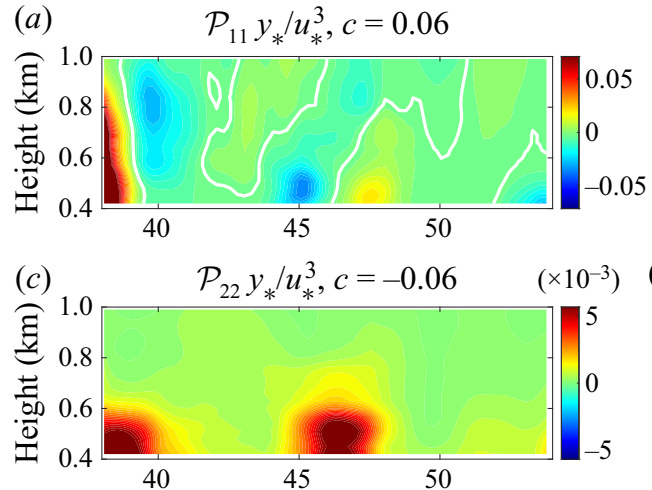

(e)

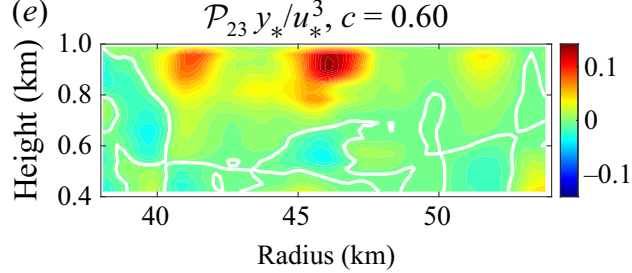

(b) $\quad \mathcal{P}_{12} y_{*} / u_{*}^{3}, c=-0.04$

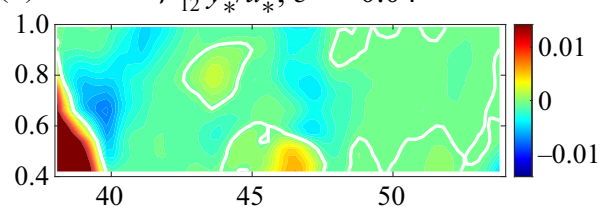

(d) $\quad \mathcal{P}_{13} y_{*} / u_{*}^{3}, c=0.82$
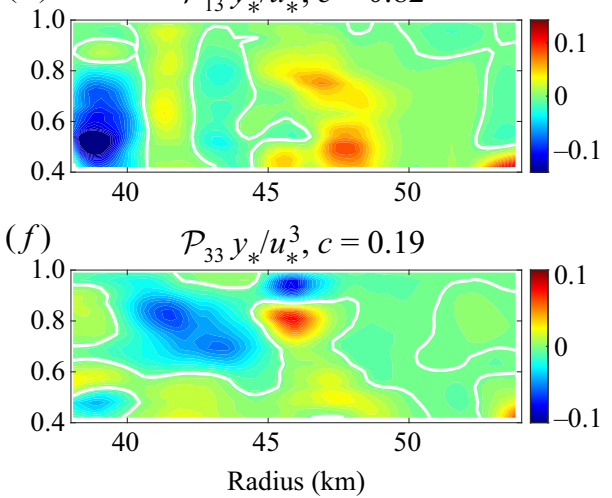

Figure 6. The components of the total SFS energy for the 1910-1920 UTC pass. The symmetric terms are not shown for brevity. The mean correlation $(c)$ between each component and the total energy $(\mathcal{P})$ is shown in each panel title. The white contour identifies where each component is zero.

Of these two, the larger influence comes from the radial-vertical interactions, which comprise the secondary circulations around the turbulent eddies shown in figure 4 .

The components of $\tilde{\bar{S}}$ are shown in figure 7 . The magnitude of the vertical shear of the radial and tangential velocities generally dominate the other shear terms, which leads to larger values of $\widetilde{S_{13}}$ and $\widetilde{S_{23}}$ relative to other strain rate tensor components. However, these two components generally have the same sign throughout the region we are considering. Additionally, the total range of values of $\tilde{\bar{S}}_{13}$ and $\tilde{\bar{S}}_{23}$ is small relative to their $\tau$ counterparts. This means that $\tilde{\bar{S}}_{13}$ and $\tilde{\bar{S}}_{23}$ generally serve to give more weight to the corresponding terms of $\tau$ in the product $\tau_{i j} \overline{S_{i j}}$, and we would expect to see a large correlation between $\mathcal{P}$ and both $\tau_{13}$ and $\tau_{23}$. Physically, this reflects the strong vertical 

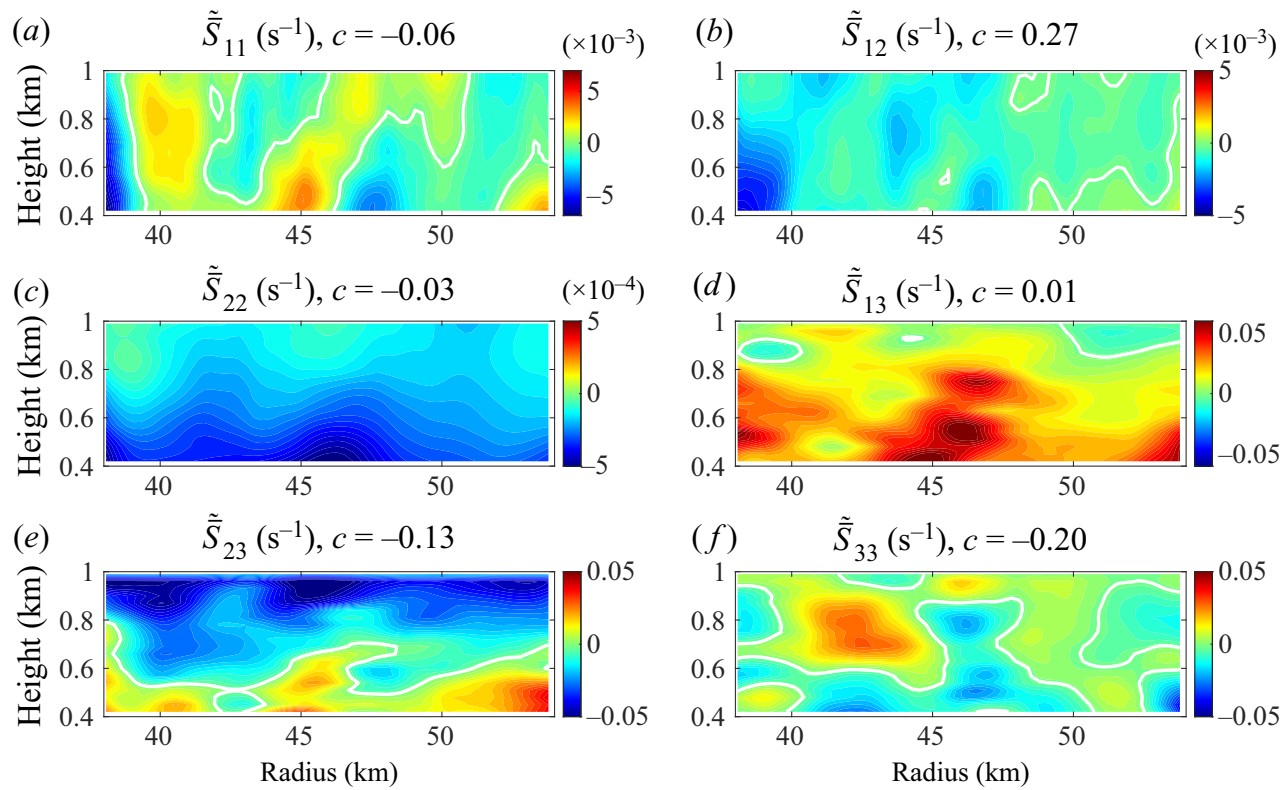

Figure 7. As in figure 6, except for the strain rate tensor of the 1910-1920 UTC pass.

and radial gradients created by the boundary layer eddies. In particular, the secondary circulations around the eddies create favourable conditions for upscale energy transfer.

Figure 8 facilitates an efficient comparison of the $L, R$ and $C$ tensors and shows that the $\tau_{13}$ and $\tau_{23}$ are indeed the most highly correlated with $\mathcal{P}$. While the Reynolds terms generally have a larger magnitude than the other two tensors, none of the tensors can be considered negligible. The vertically coherent structures in the two Reynolds terms $R_{13}$ and $R_{23}$ and the two cross-terms $C_{13}$ and $C_{23}$ dominate the structure of the corresponding $\tau$ components. This structure translates through to $\mathcal{P}$ due to the aforementioned strain rate tensor components weighting $\tau_{13}$ and $\tau_{23}$ more heavily.

The sign of $\mathcal{P}$ is largely determined by the sign of the vertical velocity field. Since $\tilde{\bar{S}}_{13}$ $\left(\tilde{\bar{S}}_{23}\right)$ is generally positive (negative) in the region examined here, the sign of the SFS energy comes from the flux tensor. The sign of the flux tensor generally corresponds to the sign of the vertical velocity field since the radial velocity is nearly uniformly negative and the tangential velocity is uniformly positive. As can be seen in figure 4, regions of backscatter (forwardscatter) occur where the vertical velocity field transitions from positive to negative (negative to positive). Figure 9 shows the radial shear of the azimuthally averaged vertical velocity field which helps confirm the collocation of the vertical velocity transition regions with the production of SFS energy.

A filter length scale sensitivity test was performed on all flight passes to study whether the dominant oscillatory structure persists when using different length scales in the filter $G$. The results of this test showed that the structure of $\mathcal{P}$ is essentially independent of the filter length scale for length scales between 1.5 and $3 \mathrm{~km}$. At larger filter length scales, the regions with stronger forwardscatter or backscatter steadily grow outwards into the adjacent regions with weaker magnitudes. At smaller length scales, noise begins to mottle the vertical coherence of the forwardscatter and backscatter regions. Again, the $2 \mathrm{~km}$ 


\section{S. Sroka and S.R. Guimond}
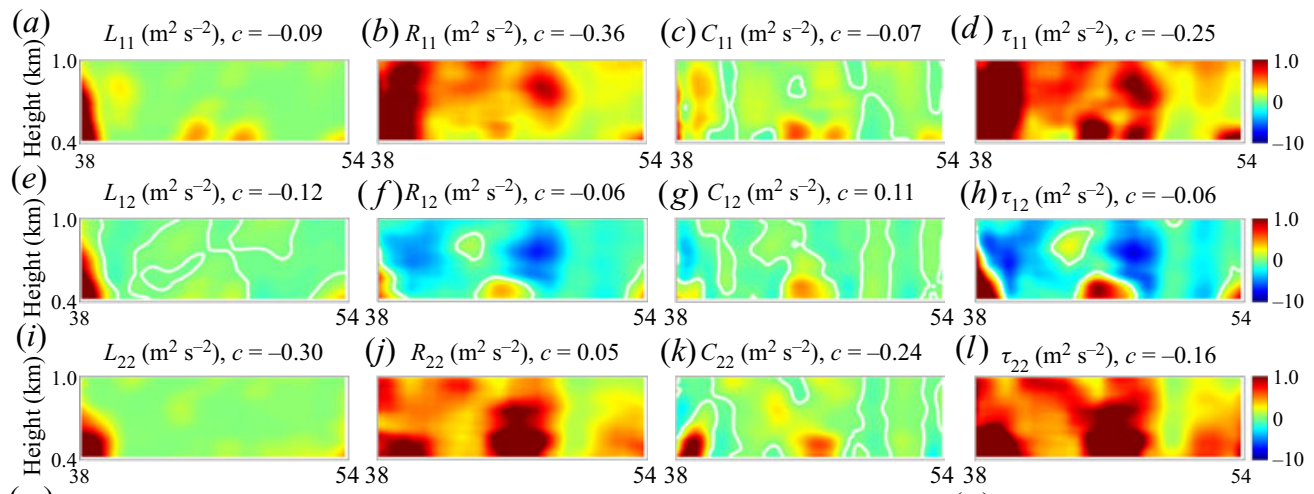

(k) $C_{22}\left(\mathrm{~m}^{2} \mathrm{~s}^{-2}\right), c=-0.24 \quad(l) \tau_{22}\left(\mathrm{~m}^{2} \mathrm{~s}^{-2}\right), c=-0.16$
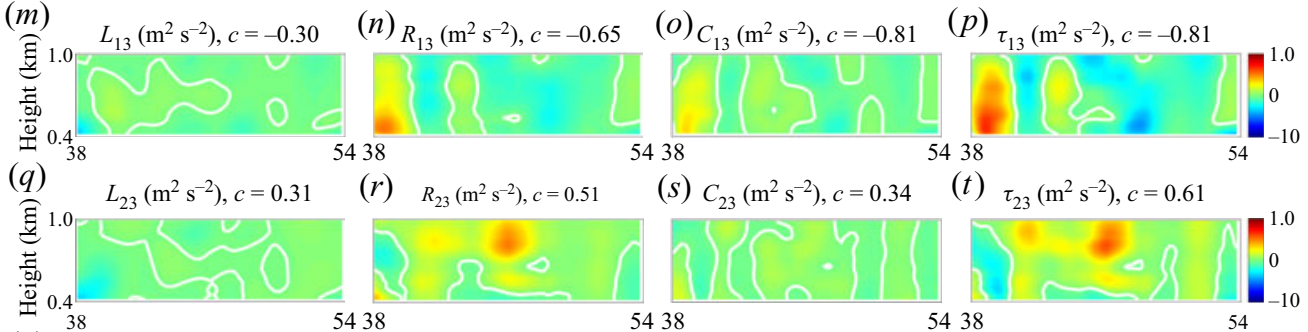

(r) $R_{23}\left(\mathrm{~m}^{2} \mathrm{~s}^{-2}\right), c=0.51$

(s) $C_{23}\left(\mathrm{~m}^{2} \mathrm{~s}^{-2}\right), c=0.34$

(t) $\tau_{23}\left(\mathrm{~m}^{2} \mathrm{~s}^{-2}\right), c=0.61$
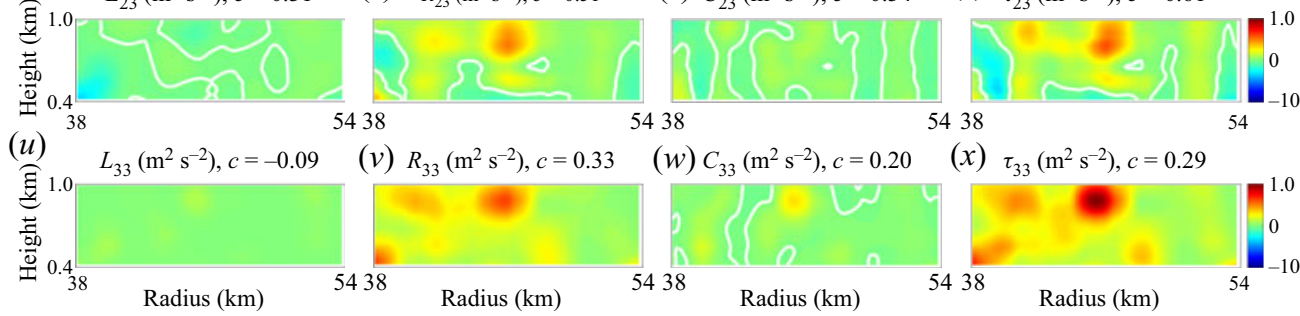

(v) $R_{33}\left(\mathrm{~m}^{2} \mathrm{~s}^{-2}\right), c=0.33$

(w) $C_{33}\left(\mathrm{~m}^{2} \mathrm{~s}^{-2}\right), c=0.20$

(x) $\tau_{33}\left(\mathrm{~m}^{2} \mathrm{~s}^{-2}\right), c=0.29$
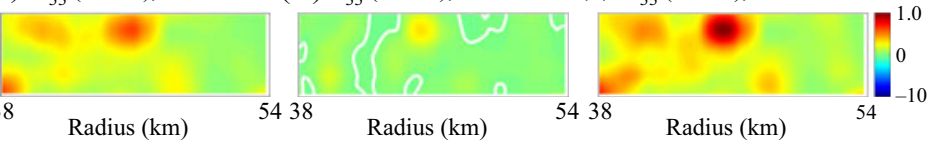

Figure 8. The components of the fluxes for the 1910-1920 UTC pass; the components of the Leonard, Reynolds and cross-term stresses are shown in the first, second and third columns, respectively. The fourth column shows the total flux tensor, which is the sum of the first three terms in each row. Again, the correlation coefficient $c$ is between each component and the total production. Each plot is over the same radial and vertical range, and the colour bar is uniform across all plots. The zero contour for each plotted component is shown in white where applicable.

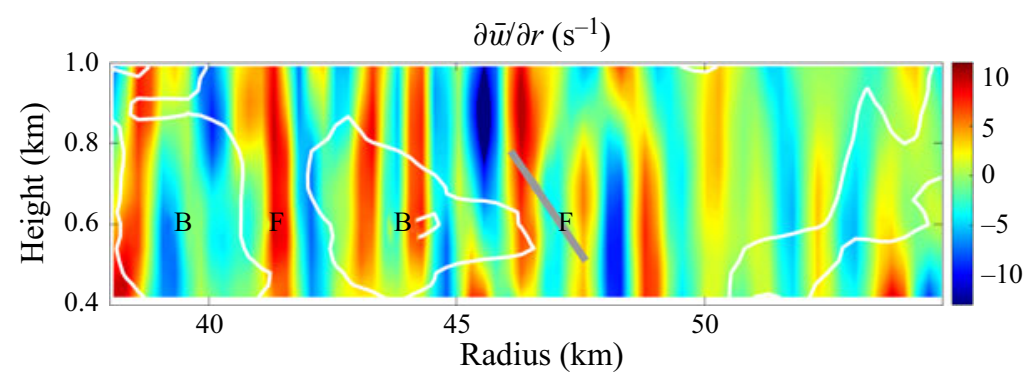

Figure 9. The radial shear of the azimuthally averaged vertical velocity for the 1910-1920 UTC pass. The grey line links the peak locations of the tilted forwardscatter, which both align with positive regions of $\partial \bar{w} / \partial r$. The other details on the figure are the same as in figure 5.

length scale used here is optimal for extending the implications of this analysis to current numerical simulations of hurricanes. 


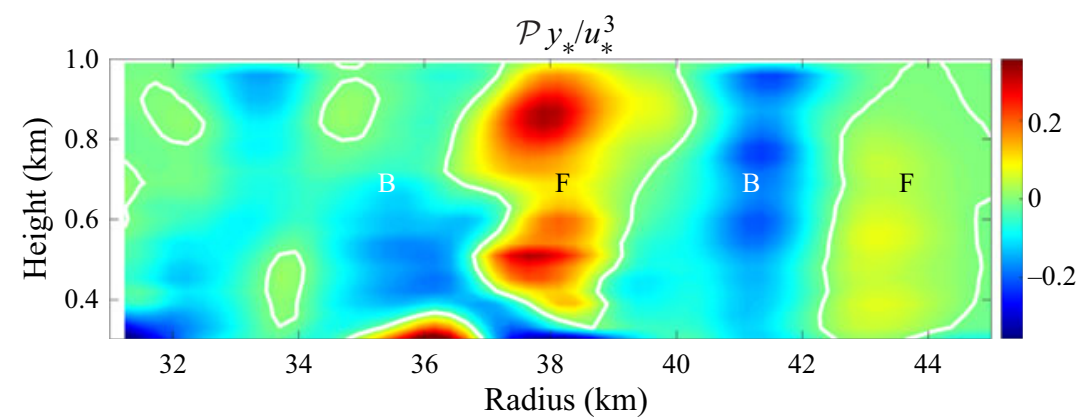

Figure 10. As in figure 5, except for the 2030-2040 UTC pass.
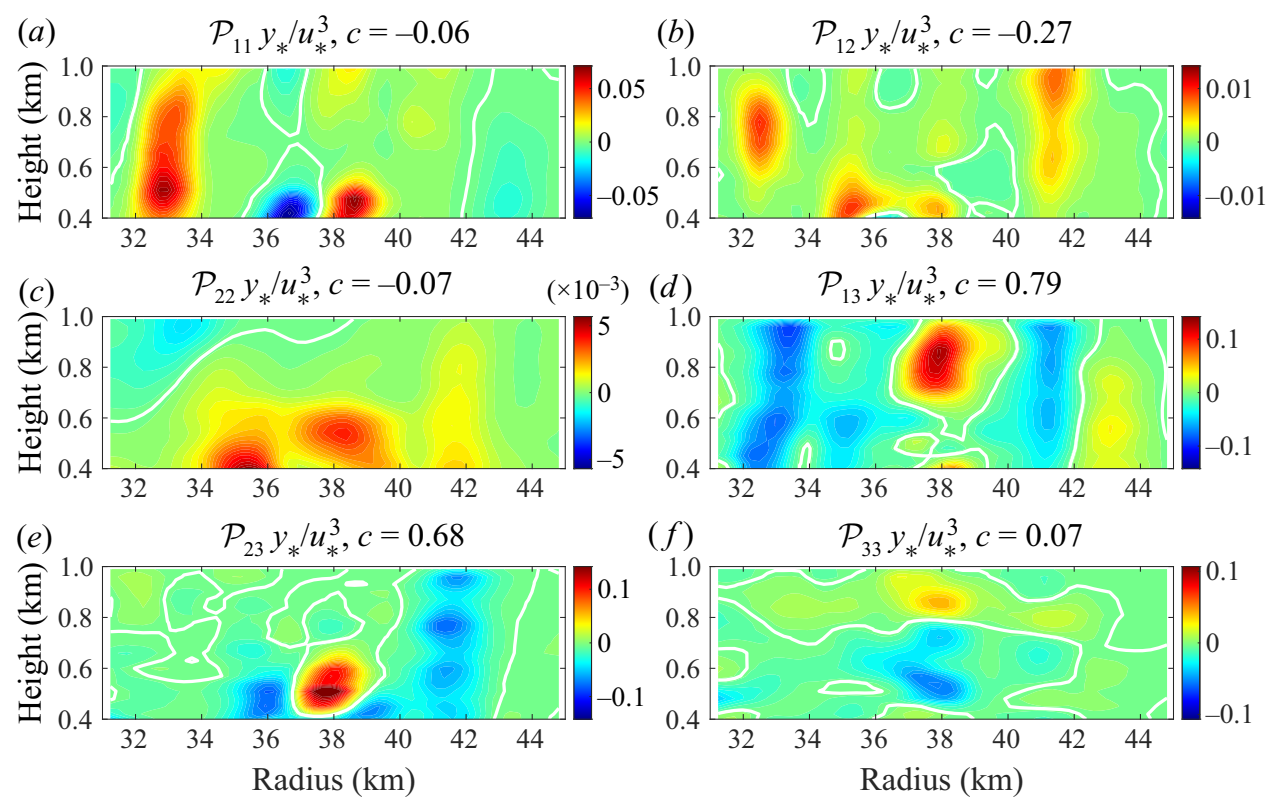

Figure 11. As in figure 6, except for the 2030-2040 UTC pass.

\subsection{September 22 2030-2040 UTC pass}

This radial leg sampled the southwestern portion of the storm that also displayed an inner and outer eyewall structure along with several fine-scale bands of radar reflectivity similar to the 1910-1920 UTC pass (see figures 4 and 8 in G18b). Also similar to the 1910-1920 UTC pass is the oscillating pattern of forwardscatter and backscatter with wavelengths of approximately $5 \mathrm{~km}$ shown in figure 10 . The most prominent regions of scatter are located in the $36-44 \mathrm{~km}$ radial band. This region contains the inner edge of the intensifying outer eyewall and has strong, turbulent eddies (G18b).

Again, the total production of SFS energy is governed largely by the $\mathcal{P}_{13}$ and $\mathcal{P}_{23}$ terms with correlation coefficients of 0.79 and 0.68 , respectively (figure 11 ). In particular, the structure of the $\mathcal{P}_{13}$ component is very consistent with the locations of the forwardscatter and backscatter regions shown in the $\mathcal{P}$ field in figure 10. As described in the analysis of the 1910-1920 UTC pass, the vertical flux of radial momentum is 


\section{S. Sroka and S.R. Guimond}
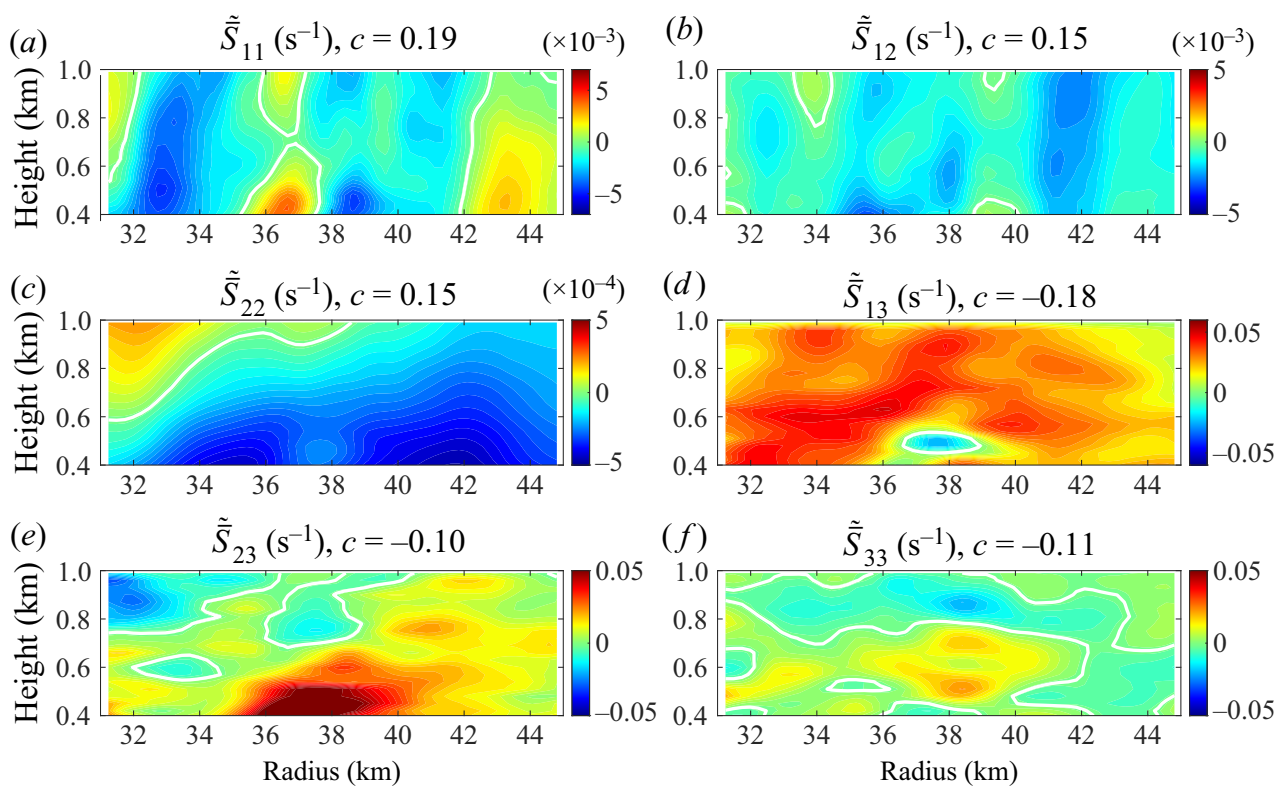

Figure 12. As in figure 7, except for the 2030-2040 UTC pass.

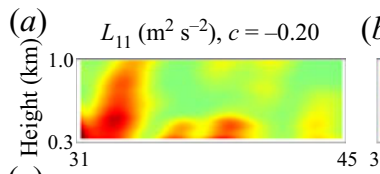

(b) $R_{11}\left(\mathrm{~m}^{2} \mathrm{~s}^{-2}\right), c=0.17$

(c) $C_{11}\left(\mathrm{~m}^{2} \mathrm{~s}^{-2}\right), c=0.11 \quad(d) \tau_{11}\left(\mathrm{~m}^{2} \mathrm{~s}^{-2}\right), c=0.07$
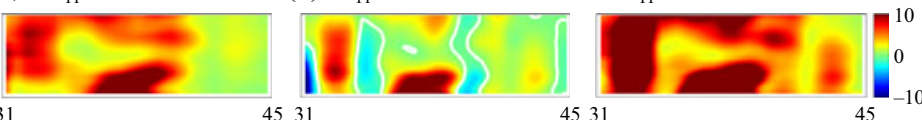

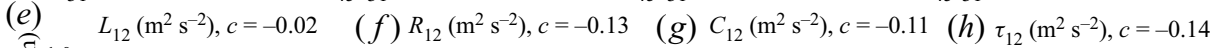
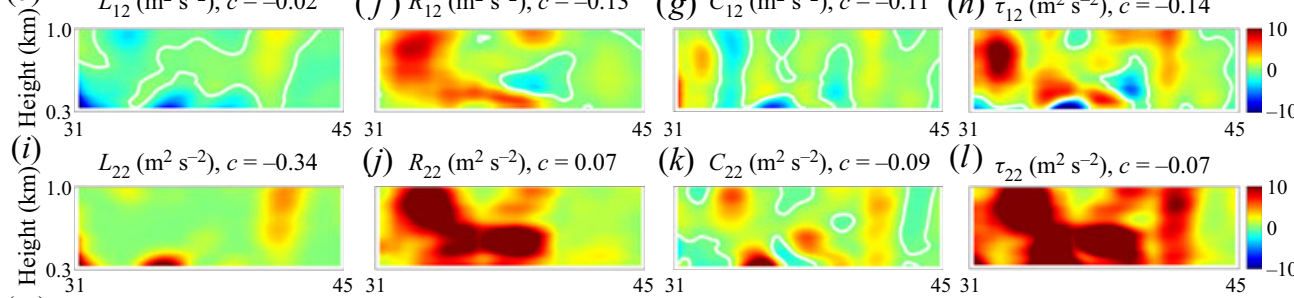

$\begin{array}{lll}\text { (j) } R_{22}\left(\mathrm{~m}^{2} \mathrm{~s}^{-2}\right), c=0.07 & \text { (k) } C_{22}\left(\mathrm{~m}^{2} \mathrm{~s}^{-2}\right), c=-0.09 & (l) \tau_{22}\left(\mathrm{~m}^{2} \mathrm{~s}^{-2}\right), c=-0.07\end{array}$
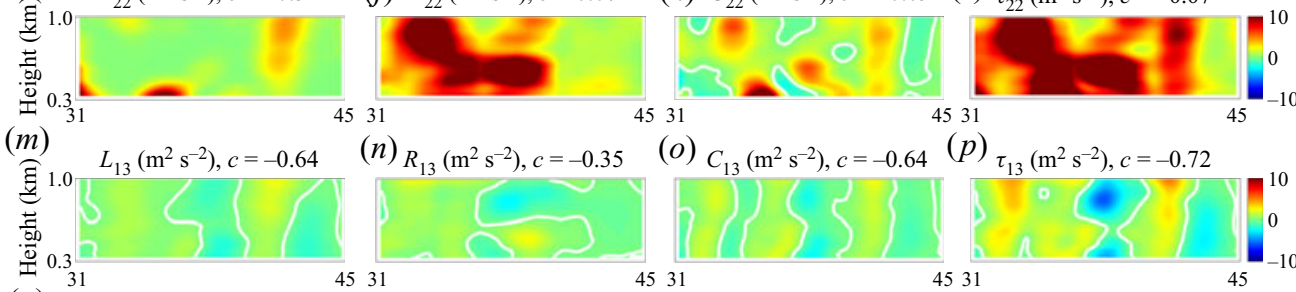

o) $C_{13}\left(\mathrm{~m}^{2} \mathrm{~s}^{-2}\right), c=-0.64(p) \tau_{13}\left(\mathrm{~m}^{2} \mathrm{~s}^{-2}\right), c=-0.72$
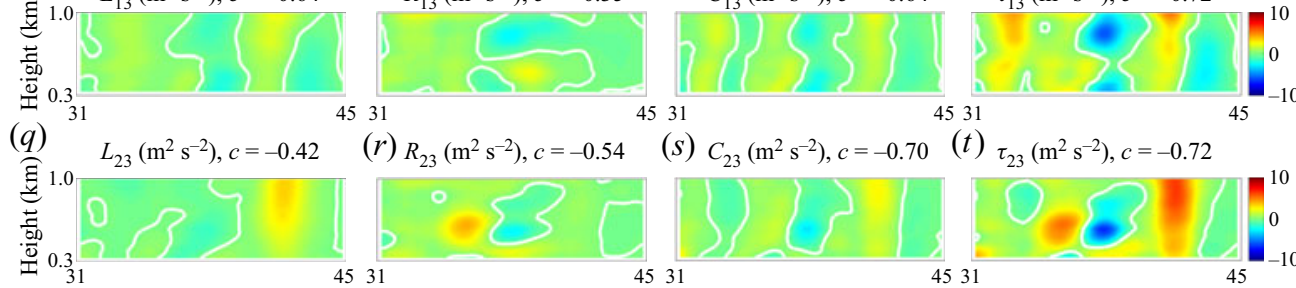

$(s) C_{23}\left(\mathrm{~m}^{2} \mathrm{~s}^{-2}\right), c=0.70$

(t) $\tau_{23}\left(\mathrm{~m}^{2} \mathrm{~s}^{-2}\right), c=-0.72$
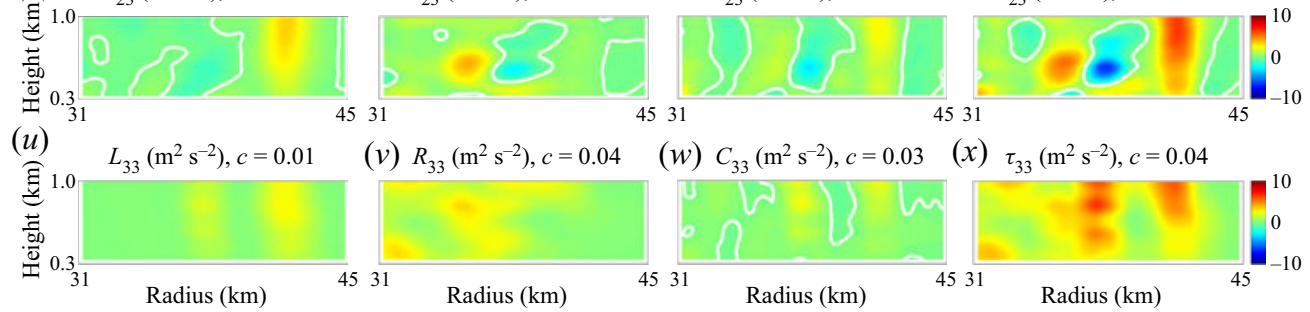

Figure 13. As in figure 8, except for the 2030-2040 UTC pass. 


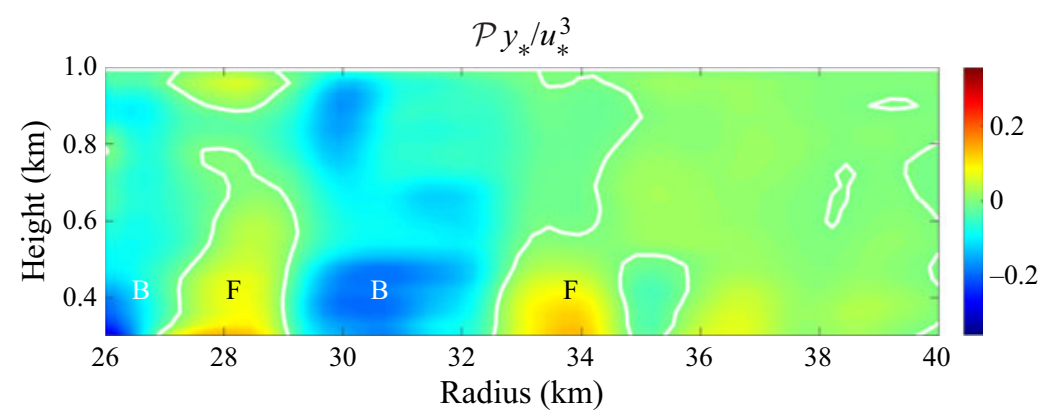

Figure 14. As in figure 5, except for the 2050-2100 UTC pass.

physically connected to the secondary circulation in the turbulent eddies, and the vertical velocities in the ascending and descending branches determine the sign of the energy transfer. The dominant terms in the strain rate tensor, shown in figure 12 , are $\tilde{\bar{S}}_{13}$ and $\tilde{\bar{S}}_{23}$; both nearly have the same sign throughout the domain and a small range of values compared to their $\tau$ counterparts. Again, this means that they effectively serve as weights which lead to $\mathcal{P}_{13}$ and $\mathcal{P}_{23}$ being highly correlated with $\mathcal{P}$, as shown in figure 13 . In this pass, the alternating, vertically coherent pattern in $C_{13}$ and $C_{23}$ shown in figure 13 is particularly prominent, though the corresponding Reynolds and Leonard terms also exhibit a discernible oscillation. Collectively these three tensors sum to a well-defined, vertically coherent pattern in the two terms $\tau_{13}$ and $\tau_{23}$, which dominate $\mathcal{P}$. The oscillations are controlled by the vertical velocity field and associated with vertical fluxes of radial and tangential momentum in the turbulent eddies.

\subsection{September 23 2050-2100 UTC pass}

This radial leg sampled the merging of the inner and outer eyewall in the northern portion of the storm (see figures 10 and 12 in G18b). Similar to the other two passes, figure 14 shows the total SFS energy production for this pass has alternating regions of backscatter and forwardscatter in the radial region where intense turbulent eddies are found. However, this pass observes the merging of the inner and outer eyewall where there is increased vertical wind shear $(\mathrm{G} 18 \mathrm{~b})$. The largest values of total energy production occur from approximately $0.5 \mathrm{~km}$ height and below, with backscatter showing larger values than forwardscatter at inner radii. There is almost no backscatter found radially outward of the approximately $35 \mathrm{~km}$ radius where the turbulent eddy activity is weaker.

In this pass, the total energy production is mostly controlled by the $\mathcal{P}_{13}$ term, with more modest contributions from $\mathcal{P}_{23}$ as shown in figure 15 . The strain rate term $\tilde{\bar{S}}_{13}$ is larger than $\tilde{\bar{S}}_{23}$ as shown in figure 16 . This leads to the greater weighting of $\tau_{13}$ in the total production term compared with $\tau_{23}$. In this pass, each of the three flux terms which compose $\tau_{13}$ and $\tau_{23}$, shown in figure 17 , have similar magnitudes and oscillating structures. However, as in the other passes, the Reynolds term $R_{13}$ and the cross-term $C_{13}$ appear to exert the biggest influence on $\tau_{13}$ and combine to produce a deep region of backscatter near $r=30 \mathrm{~km}$. These results also show that interactions among SFS motions (captured by the Reynolds stress) and the direct interaction of SFS motions with the resolved-scale motions (captured by the cross-term stress) are doing most of the work in the energy transfer. 


\section{S. Sroka and S.R. Guimond}
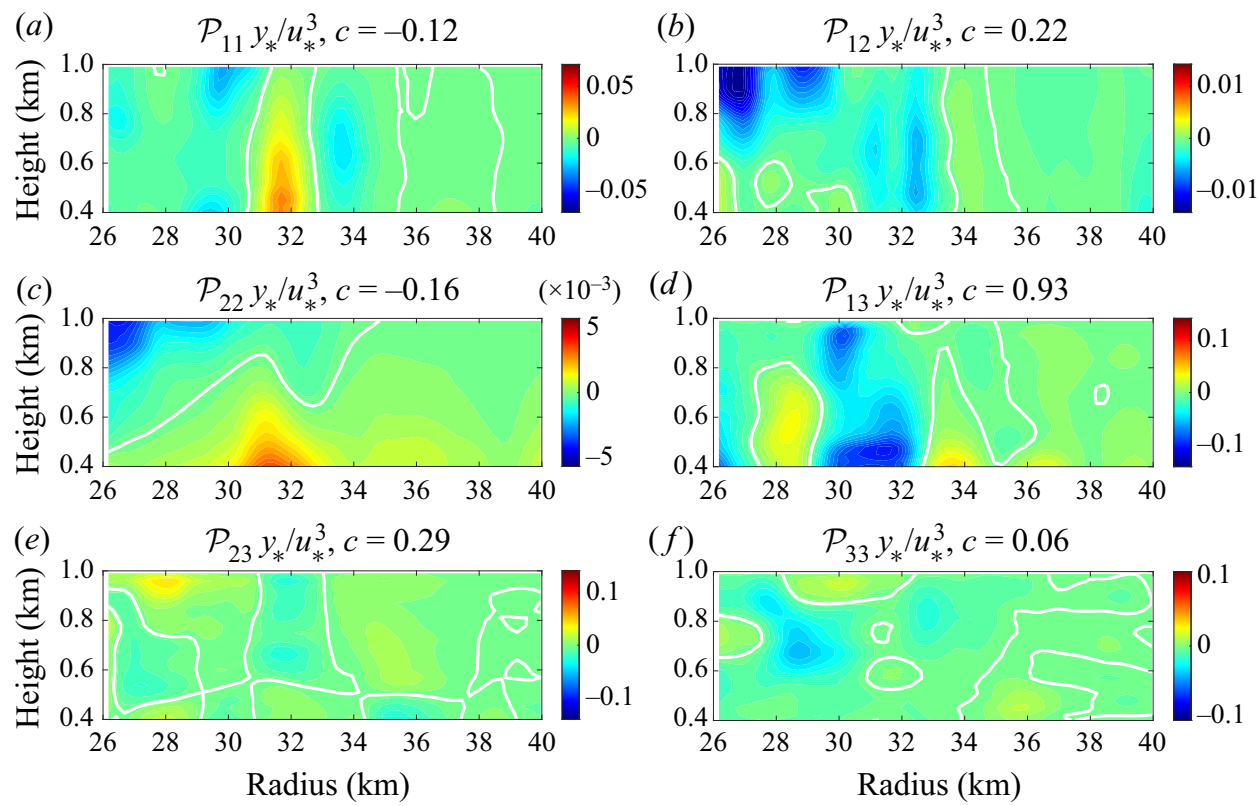

Figure 15. As in figure 6, except for the 2050-2100 UTC pass.
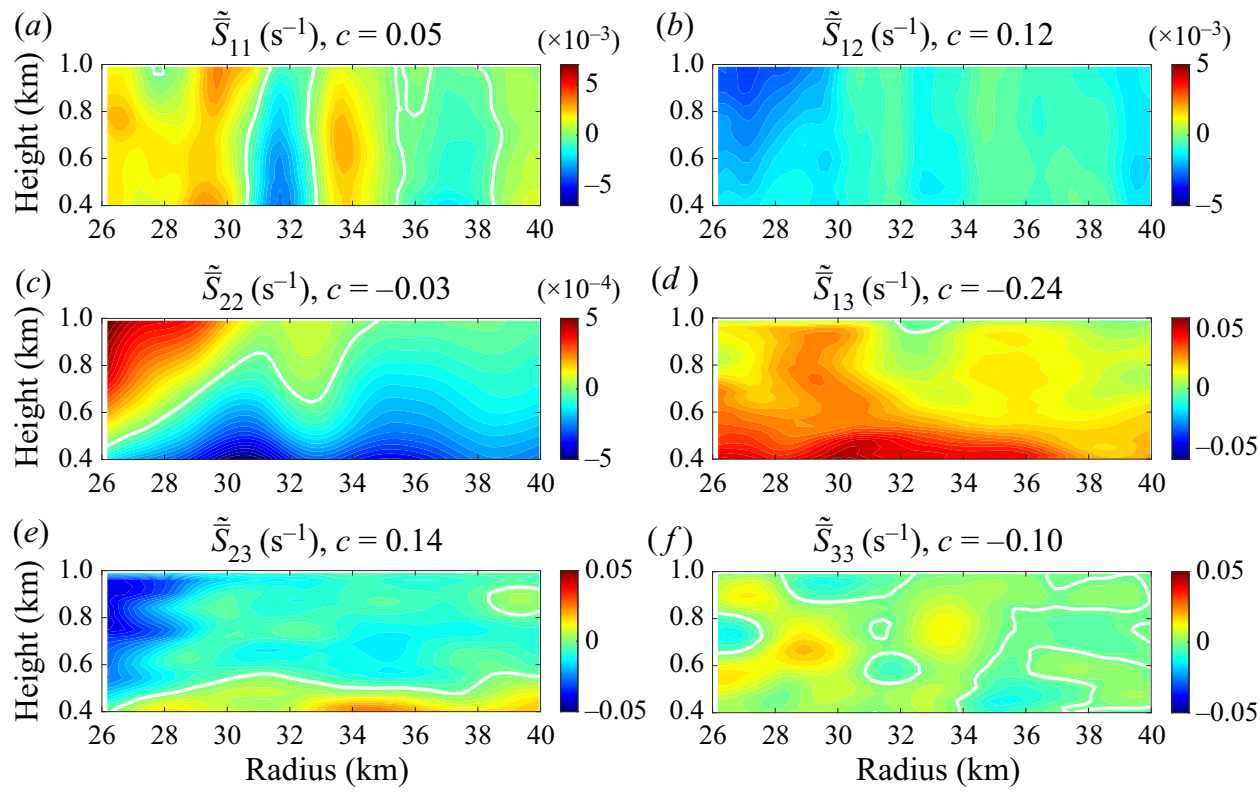

Figure 16. As in figure 7, except for the 2050-2100 UTC pass.

\section{Impacts on the KE budget}

Now that we have shown the detailed structure of the SFS energy production and how the regions of forwardscatter and backscatter are produced, it is natural to examine the role of this energy transfer in the full KE budget. The resolved-scale KE equation (2.6) is analysed here and the IWRAP wind fields can be used to calculate all terms except 

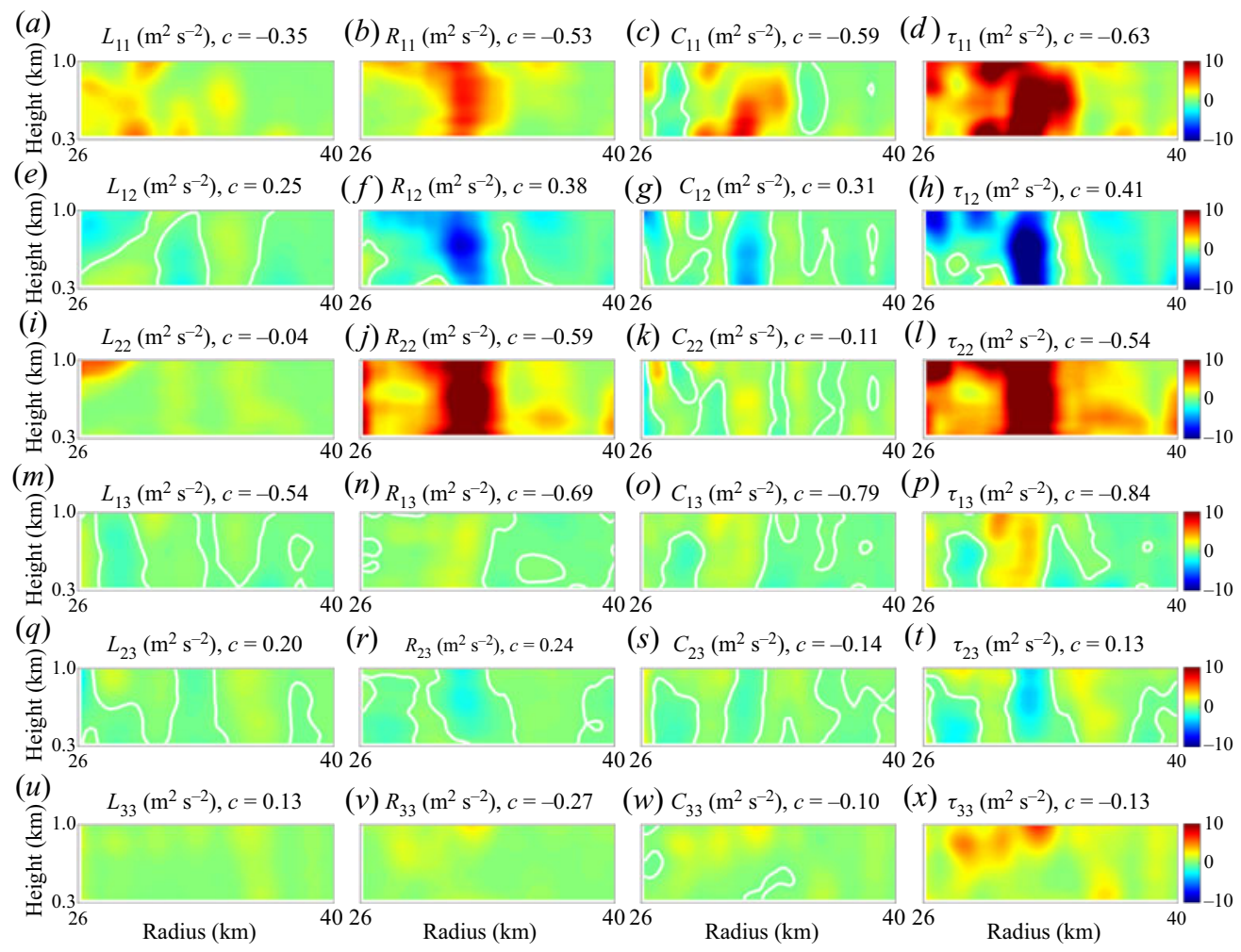

Figure 17. As in figure 8, except for the 2050-2100 UTC pass.

those involving pressure, density and the time tendency. These quantities are very difficult to measure accurately in a hurricane, especially in the turbulent boundary layer, but reasonable estimates can be made using ancillary data.

The radial and vertical gradients of pressure are estimated using in situ data collected from research aircraft. Marks et al. (2008) reported on aircraft flight-level measurements of pressure in the boundary layer of a strong hurricane with a similar intensity to the case analysed here at a height of approximately $450 \mathrm{~m}$. They found filtered radial pressure gradients of approximately $0.04 \mathrm{~Pa} \mathrm{~m}^{-1}$ at a distance of 2.5 times the radius of maximum wind, which is a radial location similar to the regions presented in this paper. Vertical profiles of pressure measured from dropsondes in Hurricane Rita (2005) during the passes analysed above reveal vertical pressure gradients of about $-10 \mathrm{~Pa} \mathrm{~m}^{-1}$ in the boundary layer, which is very close to hydrostatic balance. The dropsondes do not fall strictly in a vertical profile, but move in the tangential direction as well, due to the strong tangential winds in the boundary layer. However, the pressure does not vary much in the tangential direction so this is a minor concern. These estimates of the pressure gradient are applied uniformly throughout the boundary layer. While there will be some error in this assumption, the spatial gradients of pressure are much smaller than those of the wind field and the vast majority of the total field is described by gradient wind and hydrostatic balance. Furthermore, the density has small variability in the horizontal dimensions and we use a standard atmosphere vertical profile to approximate this variable in the budgets. Using these estimates and the IWRAP wind fields, we calculate the time tendency of the $\mathrm{KE}$ as a residual. 


\section{S. Sroka and S.R. Guimond}

1910, KE budget terms

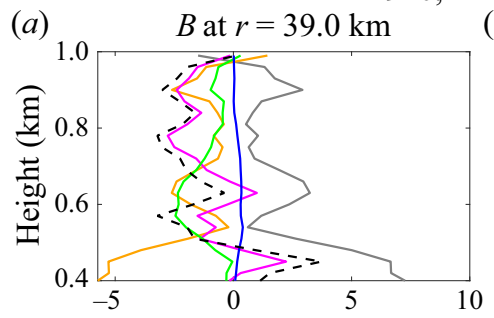

(b)

(b) $\quad F$ at $r=47.0 \mathrm{~km}$
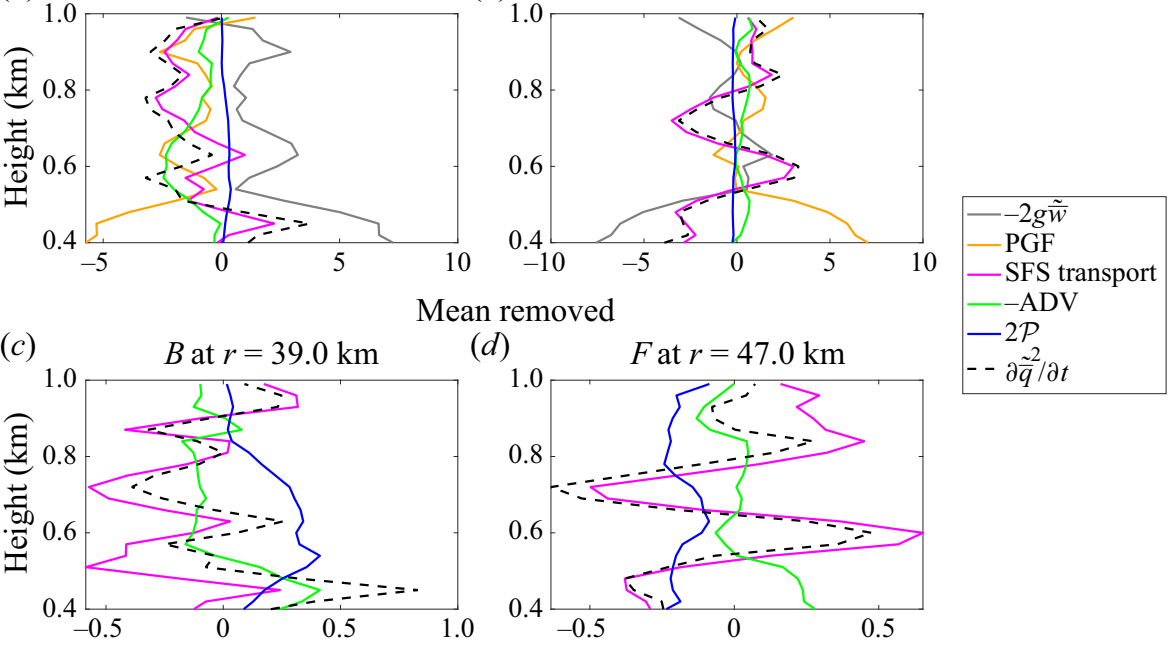

Figure 18. The KE budget terms for the 1910-1920 UTC pass. All profiles have been normalized by $u_{*}^{3} / y_{*}$.

In the following figures, we have averaged each budget contribution over the radial regions with the largest values of backscatter and forwardscatter because our goal is to estimate the impacts of this SFS energy transfer on the larger-scale flow. The top two plots in figure 18 show the resolved-scale KE budgets for the 1910 UTC pass focusing on the backscatter centred at $r=39 \mathrm{~km}$ and the forwardscatter region centred at $r=47 \mathrm{~km}$. In the backscatter region, the largest terms are the following: the gravitational term, the total pressure term (which is dominated by the vertical advection of pressure), the total advection of $\mathrm{KE}$ and the stress tensor flux divergence (which represents the transport of SFS motions). The gravitational and pressure terms are nearly equal and opposite, which reflects the strong constraint of hydrostatic balance. As a result, the SFS transport and total advection terms control most of the variability in the time tendency, which generally predicts a reduction of resolved-scale KE over the HBL depth. The backscatter term, which is a source of $\mathrm{KE}$, has a small contribution to the resolved-scale KE budget. In the forwardscatter region, a similar picture emerges with the nearly balanced gravitational and pressure contributions. The SFS transport term controls the sign of the KE time tendency, which oscillates about the zero line throughout the depth of the HBL, while the advection term provides a uniformly positive contribution. The forwardscatter magnitude is small and only has a minor effect on the resolved-scale KE here.

It is very important to note that the resolved-scale KE budget terms include the effects of the mean flow, which are very large in an intense hurricane such as Rita during this time period. Therefore, it is not surprising that the SFS energy transfer, which represents scales of $2 \mathrm{~km}$ and below, has a small direct impact on the resolved-scale KE. In order to examine the effects of the SFS energy transfer on the eddy-scale KE (recall the KE spectrum in figure 3), the mean flow in the $r-z$ plane is removed from the velocity variables. In addition, a few simplifications of the KE equation can be made. Smith \& Montgomery (2008) describe how the vertical gradient of perturbation pressure can be neglected in the HBL through scale analysis of the governing equations, which is also supported by the close hydrostatic balance calculated above. Marks et al. (2008) show very small radial gradients of perturbation pressure in the outer regions of the HBL, which is applicable to the regions analysed here. Based on this information, we neglect the perturbation pressure 


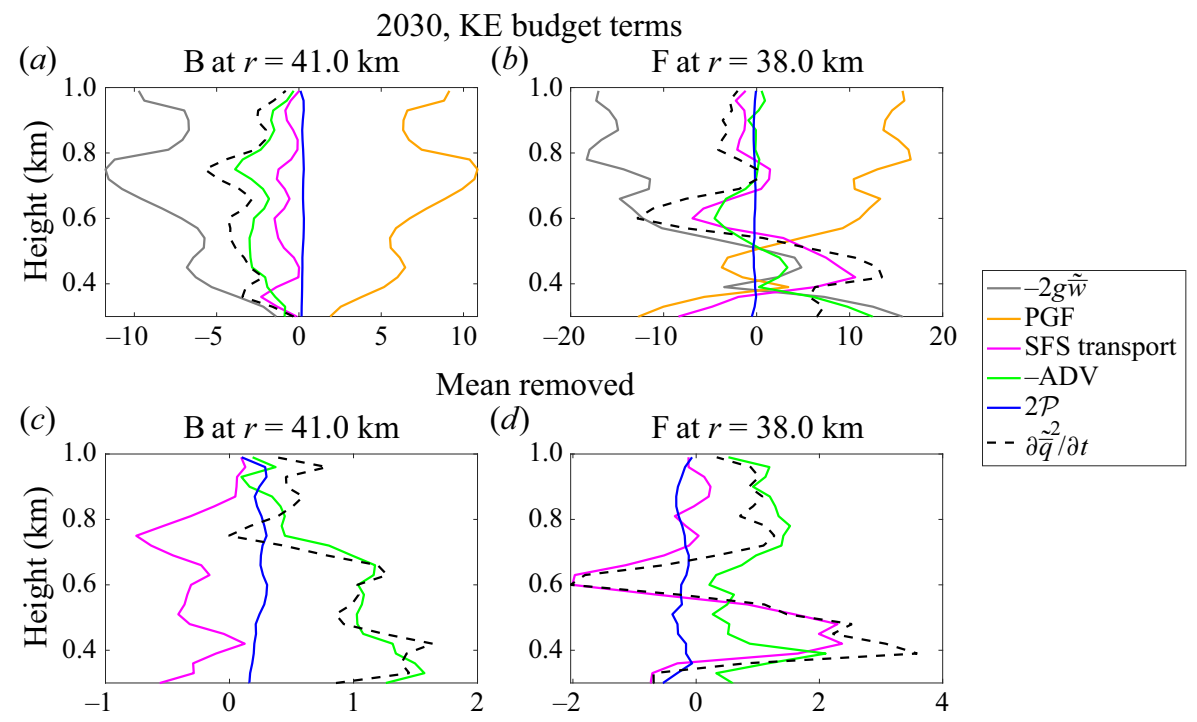

Figure 19. As in figure 18 except for the 2030-2040 UTC pass.

term in our eddy-scale budget. Furthermore, Zhang et al. (2009) and others have shown that the HBL is typically neutrally buoyant, which allows the neglect of the perturbation gravitational (buoyancy) term.

Panels $(c, d)$ in figure 18 show the eddy-scale KE budgets for the 1910 UTC pass for the same radial regions as the resolved-scale KE budgets. The backscatter and forwardscatter terms have a significant contribution to the eddy-scale KE budget in these regions with values that are of the same order as the other terms. When averaged over height, the backscatter and forwardscatter terms contribute between $30 \%$ and $40 \%$, respectively, to the local time tendency of eddy-scale KE. For the weaker regions of scatter shown in figure 5, the backscatter (near $r=44 \mathrm{~km}$ ) and forwardscatter (near $r=41 \mathrm{~km}$ ) terms contribute between $6 \%$ and $50 \%$, respectively, to the eddy-scale KE (not shown). The larger contribution from forwardscatter in this region is due to smaller values of the advection term. The structure of the time tendency term is similar to that of the resolved-scale KE budget, but now the SFS energy transfer terms are able to move the time tendency in either the positive or negative direction. For example, in the backscatter region near $r=39 \mathrm{~km}$, the positive SFS energy transfer has helped to create regions of increasing $\mathrm{KE}$, which does not occur in the resolved-scale KE budget.

Figure 19 shows the resolved-scale and eddy-scale KE budgets for the 2030 UTC pass focusing on the region of backscatter centred at $r=41 \mathrm{~km}$ and the region of forwardscatter centred at $r=38 \mathrm{~km}$. The results are similar to those from the 1910 UTC pass. The production of SFS energy plays a small role in the resolved-scale dynamics, but the eddy-scale KE budgets show that the SFS energy transfer does have a meaningful impact on the eddy-scale motions. Averaging over height, the backscatter and forwardscatter terms contribute between $16 \%$ and $32 \%$, respectively, to the local time tendency of eddy-scale KE. In the weaker regions of scatter shown in figure 10, the backscatter (near $r=36 \mathrm{~km}$ ) and forwardscatter (near $r=43 \mathrm{~km}$ ) terms contribute between $15 \%$ and $2 \%$, respectively, to the eddy-scale KE (not shown).

The budget analyses described above demonstrate that the SFS energy transfer can have a substantial impact on the larger-scale eddy motions. This indicates that the coherent turbulent eddies, and their associated SFS energy transfer, will affect the HBL and 


\section{S. Sroka and S.R. Guimond}

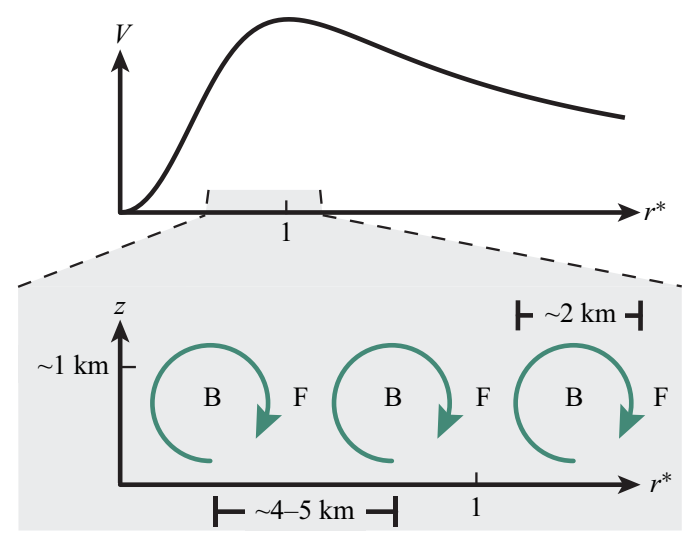

Figure 20. The upper panel shows a typical azimuthal mean wind profile in a mature hurricane where the radial coordinate is normalized by the radius of maximum wind. The lower panel shows a conceptual diagram of the observed secondary circulations associated with boundary layer eddies and the phasing of peaks in forwardscatter (F) and backscatter (B).

vortex dynamics primarily through wave-wave nonlinear interactions, which can feed energy into the mean flow through wave-mean flow interactions. This pathway only considers a dynamical interpretation, but thermodynamic effects from the eddies are also anticipated. For example, enhanced mass convergence and fluxes of enthalpy from the ocean to the atmosphere in the HBL can result in increased convective activity, which will have additional feedbacks on the mean flow. It is also worthwhile to note that the material derivative of $\mathrm{KE}$ is substantial relative to other terms in both the resolved-scale and eddy-scale budgets over much of the domain, which highlights the non-conservative nature of the HBL. The SFS transport and production of SFS energy terms control the magnitude and structure of the material derivative of $\mathrm{KE}$, which is more clearly seen in the eddy-scale budgets. These terms are physically connected to the coherent turbulent eddies and therefore, regions of the HBL with prominent coherent structures are likely to contain substantial non-conservative effects. It is difficult to compare this result to prior measurement work because, to the best of the authors' knowledge, this is the first study to examine all terms in the KE budget in the HBL. In addition, we utilize the filtering or LES approach rather than the more common Reynolds-averaged Navier-Stokes or RANS approach. In the general marine atmospheric boundary layer, most studies assume that the material derivative of turbulent KE is approximately zero (Stull 1988; Sjöblom \& Smedman 2002), which is not the case for the highly dynamic and turbulent conditions found in the HBL.

\section{Conclusions and implications}

Studying the transfer of energy in large-Reynolds-number flows, especially in the turbulent boundary layer, is important for understanding the flow physics and designing accurate sub-filter models. In this paper, we have computed and analysed the energy transfer in the HBL using turbulence measurements from the IWRAP airborne Doppler radar collected in Hurricane Rita (2005) during peak intensity. The following results were found from this analysis:

(i) Strong regions of forwardscatter and backscatter are found in the hurricane boundary layer with an organized, vertically coherent structure and radial wavelengths of $\sim 4-5 \mathrm{~km}$. 


\section{Organized backscatter in the hurricane boundary layer}

(ii) The oscillations in the SFS energy transfer are associated with coherent turbulent eddies that have wavelengths of $\sim 2 \mathrm{~km}$, and are possibly boundary layer roll vortices, identified in prior work (Guimond et al. 2018b). The region between two eddies is favourable for forwardscatter.

(iii) The secondary circulations (in the radius-height plane) contained in the coherent turbulent eddies are driving the oscillation in the energy transfer with the ascending and descending branches controlling the sign of the SFS fluxes, which determines the sign of the energy transfer.

(iv) The largest components of strain are generally $\widetilde{S_{13}}$ and $\widetilde{S_{23}}$, and since these fields generally have a uniform sign and relatively homogeneous structure compared with the corresponding $\tau$ terms, these components of strain serve to weight $\tau_{13}$ and $\tau_{23}$ more heavily in the total production calculation. Therefore, the oscillatory structure in $\tau_{13}$ and $\tau_{23}$ predominate in the total production field as shown by their large correlation coefficients with the total production (on average $79 \%$ for $\tau_{13}$ and $49 \%$ for $\tau_{23}$ across all passes). The breakdown of the fluxes into Leonard, Reynolds and cross-term stresses shows that the Reynolds and cross-term stresses are generally the most influential components in the flux tensor for filter widths of $2 \mathrm{~km}$. While the magnitude of the energy transfer and components change with different filter widths, the identified oscillatory structure and presence of significant backscatter are robust.

(v) The role of the SFS energy transfer in the HBL dynamics is estimated by computing the resolved-scale and eddy-scale KE budgets. The resolved-scale budget, which includes a significant contribution from the mean flow, is not greatly impacted by the production of SFS energy. After removing the mean flow to estimate the eddy-scale budget, we find the centres of peak SFS energy transfer can have a substantial impact on the larger-scale flow. Specifically, the SFS energy transfer term is consistently of the same order as the other budget terms and, when averaged over height, the contributions vary between $16 \%$ and $40 \%$ across all data collection intervals with an average of approximately $30 \%$. This implies that the primary dynamical pathway for the coherent turbulent eddies to influence the hurricane vortex is through wave-wave nonlinear interactions. In the case of backscatter, upscaling of energy to larger wavelengths can enhance the wave-mean flow forcing of the vortex in addition to anticipated thermodynamical effects not analysed here.

The results described above have several similarities to coherent structures (e.g. quasi-streamwise and hairpin vortices) in the turbulent boundary layer of simple, low-Reynolds-number flows (Robinson 1991; Piomelli et al. 1996; Adrian, Meinhart \& Tomkins 2000; Natrajan \& Christensen 2006). Natrajan \& Christensen (2006) studied hairpin vortices with particle-image velocimetry measurements in the log layer of wall turbulence. Although only two-dimensional velocity fields were analysed, the authors found that strong forwardscatter and backscatter regions were spatially coincident to individual hairpin vortices, similar to the three-dimensional turbulent eddies studied here. They also found that the most dominate forwardscatter regions were created at the interface between adjacent hairpin vortices through the interaction of Reynolds-stress-producing events. Similar vortical interactions and associated energy transfer patterns are found in this paper. However, the forwardscatter regions and magnitudes were dominant in Natrajan \& Christensen (2006) with weaker and smaller regions of backscatter around the hairpin heads, which is in contrast to the results presented here.

The conceptual diagram in figure 20 idealizes the observed secondary circulations and the approximate locations of forwardscatter and backscatter found in this study. 


\section{S. Sroka and S.R. Guimond}

Radial inflow is observed at the lowest levels in the boundary layer and alternating regions of ascending and descending motions are approximately uniformly spaced. Backscatter is observed where the flow transitions from ascending to descending and forwardscatter is observed where the flow transitions from descending to ascending. The low level radial inflow weakens with height and several passes showed radial outflow at the upper levels of the boundary layer. This overturning is pictured in figure 20, but the vertical depth of these features is uncertain. The radial extent over which these structures were observed was mostly inside the radius of maximum wind, whether that radius was located in the inner or outer eyewall region. While the azimuthal flow is significantly stronger than either the radial or vertical velocities, it is not clear how the peaks in azimuthal flow align with the peaks and troughs in the total SFS energy production.

The results of this study provide important observational estimates of the SFS energy transfer and their role in the KE budget. This is the first presentation and analysis of these calculations in the HBL. Future work will include a modelling study with idealized SFS energy forcing in the HBL to study the net impact of alternating regions of forwardscatter and backscatter on the vortex intensity and structure. Another avenue motivated by these findings is to quantify the impact of vertically coherent SFS energy regions with alternating signs, which is in contrast to studies that employ random, unstructured backscatter forcing.

Acknowledgements. The authors thank NOAA/NESDIS (P. Chang and Z. Jelenak), the University of Massachusetts Amherst Microwave Remote Sensing Laboratory (D. Fernandez, S. Frasier and J. Sapp) and Remote Sensing Solutions (J. Carswell) for their engineering and data collection efforts with IWRAP.

Funding. S.R.G. was partially supported by the NOAA Weather Program Office under grant NA19OAR4590329 directed by M. Vincent. We thank three anonymous reviewers for their constructive comments that helped to improve the clarity and quality of this paper.

Declaration of interests. The authors report no conflict of interest.

\section{Author ORCIDs.}

(1) Sydney Sroka https://orcid.org/0000-0002-8225-7568;

(D) S.R. Guimond https://orcid.org/0000-0001-7185-5629.

\section{REFERENCES}

Adrian, R.J., Meinhart, C.D. \& Tomkins, C.D. 2000 Vortex organization in the outer region of the turbulent boundary layer. J. Fluid Mech. 422, 1-54.

BRYAN, G.H. 2012 Effects of surface exchange coefficients and turbulence length scales on the intensity and structure of numerically simulated hurricanes. Mon. Weath. Rev. 140, 1125-1143.

BRYAN, G.H. \& ROTUNNO, R. 2009 The effects of small-scale turbulence on maximum hurricane intensity. In 13th Conference on Mesoscale Processes, Amer. Meteor. Soc., p. 4. https://ams.confex.com/ams/pdfpapers/ 154827.pdf.

Chong, M.S., Perry, A.E. \& CAntwell, B.J. 1990 A general classification of three-dimensional flow fields. Phys. Fluids A: Fluid Dyn. 2 (5), 765-777.

Cushman-Roisin, B. 1994 Introduction to Geophysical Fluid Dynamics. Prentice Hall.

Fernandez, D.E., Kerr, E.M., Castells, A., Carswell, J.R., Frasier, S.J., Chang, P.S., Black, P.G. \& MARKS, F.D. 2005 IWRAP: the imaging wind and rain airborne profiler for remote sensing of the ocean and the atmospheric boundary layer within tropical cyclones. IEEE Trans. Geosci. Remote Sens. 43 (8), 1775-1787.

FRANKLIN, J.L., BLACK, M.L. \& VALDE, K. 2003 Gps dropwindsonde wind profiles in hurricanes and their operational implications. Weather Forecast. 18, 32-44.

Germano, M. 1986 A proposal for a redefinition of the turbulent stresses in the filtered Navier-Stokes equations. Phys. Fluids 29 (7), 2323-2324.

Germano, M., Piomelli, U., Moin, P. \& CAвot, W.H. 1991 A dynamic subgrid-scale eddy viscosity model. Phys. Fluids A: Fluid Dyn. 3, 5-17. 


\section{Organized backscatter in the hurricane boundary layer}

Guimond, S.R., SRokA, S. \& Proztko, D. 2018a A large eddy simulation of hurricane intensification. In 33rd Conference on Hurricanes and Tropical Meteorology, Amer. Meteor. Soc., p. 17. https://ams.confex. com/ams/33HURRICANE/webprogram/Manuscript/Paper339570/large_eddy_33hurr_guimond.pdf.

Guimond, S.R., Reisner, J.M., MarRas, S. \& Giraldo, F.X. 2016 The impacts of dry dynamic cores on asymmetric hurricane intensification. J. Atmos. Sci. 73 (12), 4661-4684.

Guimond, S.R., Tian, L., Heymsfield, G.M. \& Frasier, S.J. 2014 Wind retrieval algorithms for the IWRAP and HIWRAP airborne doppler radars with applications to hurricanes. J. Atmos. Ocean. Technol. 31 (6), 1189-1215.

Guimond, S.R., ZhANG, J.A., SAPP, J.W., FrASIER, S.J. 2018b Coherent turbulence in the boundary layer of Hurricane Rita (2005) during an eyewall replacement cycle. J. Atmos. Sci. 75 (9), 3071-3093.

Ito, J., Oizumi, T. \& NiIno, H. 2017 Near-surface coherent structures explored by large eddy simulation of entire tropical cyclones. Sci. Rep. 7, 3798.

JUdT, F., CHEN, S.S. \& BERNER, J. 2016 Predictability of tropical cyclone intensity: scale-dependent forecast error growth in high-resolution stochastic kinetic-energy backscatter ensembles. Q. J. R. Meteorol. Soc. $142,43-57$.

KEPERT, J.D. 2012 Choosing a boundary layer parameterization for tropical cyclone modeling. Mon. Weath. Rev. 140, 1427-1445.

Kraichnan, R.H. 1976 Eddy viscosity in two and three dimensions. J. Atmos. Sci. 33, 1521-1536.

LEITH, C.E. 1990 Stochastic backscatter in a subgrid-scale model: plane shear mixing layer. Phys. Fluids A 2 , 297-299.

LEONARD, A. 1975 Energy cascade in large-eddy simulations of turbulent fluid flows. In Turbulent Diffusion in Environmental Pollution (ed. F. Frenkiel \& R. Munn), Advances in Geophysics, vol. 18, pp. 237-248. Elsevier. http://www.sciencedirect.com/science/article/pii/S0065268708604641

MAKIN, V.K. \& KudRYAVTSEV, V.N. 1999 Coupled sea surface-atmosphere model: 1. Wind over waves coupling. J. Geophys. Res.: Oceans 104 (C4), 7613-7623.

Marks, F.D., Black, P.G., Montgomery, M.T. \& Burpee, R.W. 2008 Structure of the eye and eyewall of hurricane hugo (1989). Mon. Weath. Rev. 136, 1237-1259.

NATRAJAN, V.K. \& CHRISTENSEN, K.T. 2006 The role of coherent structures in subgrid-scale energy transfer within the log layer of wall turbulence. Phys. Fluids 18, 0651041.

Piomelli, U., Савот, W.H., Moin, P. \& LeE, S. 1991 Subgrid-scale backscatter in turbulent and transitional flows. Phys. Fluids A: Fluid Dyn. 3 (7), 1766-1771.

Piomelli, U., YU, Y. \& ADRiAn, R.J. 1996 Subgrid-scale energy transfer and near-wall turbulence structure. Phys. Fluids 8, 215-224.

Robinson, S.K. 1991 Coherent motions in the turbulent boundary layer. Annu. Rev. Fluid Mech. 23, 601-639.

Rotunno, R., Chen, Y., Wang, W., Davis, C., Dudhia, J. \& Holland, G. 2009 Large eddy simulation of an idealized tropical cyclone. Bull. Am. Meteorol. Soc 90, 1783-1788.

Rutgersson, A. \& Sullivan, P.P. 2005 The effect of idealized water waves on the turbulence structure and kinetic energy budgets in the overlying airflow. Dyn. Atmos. Oceans 38 (3-4), 147-171.

ShutTs, G.J. 2005 A kinetic energy backscatter algorithm for use in ensemble prediction systems. $Q . J$. $R$. Meteorol. Soc. 131, 3079-3102.

SJÖBlom, A. \& SMEDMAN, A.S. 2002 The turbulent kinetic energy budget in the marine atmospheric surface layer. J. Geophys. Res.: Oceans 107 (C10), 3142.

Smith, R.K. \& Montgomery, M.T. 2008 Balanced boundary layers used in hurricane models. Q. J. R. Meteorol. Soc. 134, 1385-1395.

SMith, R.K. \& ThOMSEn, G.L. 2010 Dependence of tropical cyclone intensification on the boundary layer representation in a numerical model. Q. J. R. Meteorol. Soc. 136A, 1671-1685.

StUlL, R.B. 1988 An Introduction to Boundary Layer Meteorology. Kluwer Academic.

Sullivan, P.P., Horst, T.W., Lenschow, D.H., Moeng, C.-H. \& Weil, J.C. 2003 Structure of subfilter-scale fluxes in the atmospheric surface layer with application to large-eddy simulation modelling. J. Fluid Mech. 482, 101-139.

TENNEKES, H. \& LuMLEY, J.L. 1972 A First Course in Turbulence. MIT.

WANG, L.-H., Zhang, W.-Y., HaO, X., Huang, W.-X., Shen, L., Xu, C.-X. \& Zhang, Z. 2020 Surface wave effects on energy transfer in overlying turbulent flow. J. Fluid Mech. 893, A21.

Zhang, J.A., Drennan, W.M., Black, P.G. \& French, J.R. 2009 Turbulence structure of the hurricane boundary layer between the outer rainbands. J. Atmos. Sci. 66, 2455-2467. 